\title{
Global existence of weak solutions to the incompressible Vlasov-Navier-Stokes system coupled to convection-diffusion equations
}

\author{
Laurent Boudin, David Michel, Ayman Moussa
}

March 13, 2020

\begin{abstract}
We study the existence of global weak solutions in a three-dimensional time-dependent bounded domain for the incompressible Vlasov-NavierStokes system which is coupled with two convection-diffusion equations describing the air temperature and its water vapor mass fraction. This newly introduced model describes respiratory aerosols in the human airways when one takes into account the hygroscopic effects, also inducing the presence of extra variables in the aerosol distribution function, temperature and size. The mathematical description of these phenomena leads us to make the assumption that the initial distribution of particles does not contain arbitrarily small particles. The proof is based on a regularization and approximation strategy that we solve by deriving several energy estimates, including ones with temperature and size.
\end{abstract}

\section{Introduction}

The motion of a dispersed phase made of small particles inside a fluid can be described through a fluid-kinetic model (see [26]). The unknowns corresponding to the fluid are the usual macroscopic quantities such as the velocity and pressure while the dispersed phase is described by a distribution function. Under the socalled thin spray hypothesis, [11] proposes a model in which the fluid and kinetic equations are coupled through a drag term. It depends on the fluid unknowns as well as the distribution function and describes the energy and momentum exchanges between the two phases.

Here, we focus on the variation of the size of the particles due to the evaporation of the water they contain or, on the contrary, to the condensation of the water vapor in the air around the particle. As in [21], we consider a fluid-kinetic model that takes into account the variation of temperature of the fluid due to the transport of the particles, whose own temperature can also vary. In the kinetic part of the model, the aerosol distribution function has then not only the usual time, space and velocity variable set, but also the particle size and temperature, inducing new difficulties to be dealt with. 
The model studied in this article describes the transport and deposition of a therapeutic aerosol evolving in a Newtonian viscous incompressible fluid, the air, in the first generations of the human bronchial tree. The study of this phenomenon related to respiration began with 2] and [15] and the system we analyze in this paper was presented in [6] in a fixed domain. Numerous works have contributed to this study for several decades while taking into account various parameters and physical processes such as the compressibility or viscosity of the fluid, the transport of the particles, the interaction between the particles and the fluid or the walls, etc. But, up to our knowledge, the existence of a solution taking into account the variation of the size and temperature of the particles has not been studied yet.

Indeed, the following contributions all assume that the effects of the variation of the size of the particles are negligible. In a first contribution to the case of a compressible fluid, the local-in-time existence of classical solutions is proven in [3]. The same kind of result is obtained by 22 for the Euler-Vlasov-Boltzmann system. In [23], the authors prove the global existence of weak solutions to the compressible Navier-Stokes-Vlasov-Fokker-Planck system. The global existence of solutions around the equilibrium is studied in [9] and [20] and an asymptotic analysis is conducted in [24]. More recently, under the assumption of existence of local weak solutions, the study of a hydrodynamic limit for a system of the type Navier-Stokes-Vlasov-Fokker-Planck in the compressible case is conducted in [10.

The study of the Vlasov-Navier-Stokes system in the case of an incompressible fluid began with [1] and [17. Existence of weak solutions is proven in [5] in a periodic framework. This result is extended to a bounded domain by [27] and then to a time-dependent bounded domain by [8]. In dimension two, [16 proves the existence and stability of regular equilibria for the Vlasov-NavierStokes system, and the uniqueness of weak solutions in the whole space or a periodic domain is obtained in [18. More recently, the asymptotic behavior of the weak solutions of the Vlasov-Navier-Stokes system in a periodic framework in two or three dimensions is studied in [19].

In [13, the authors propose to simulate the variation of the particle size due to the evaporation or condensation of the water thanks to a multi-fluid model which relies on equations for the first and second moments of the distribution function. Another static point of view is assumed in 21 to tackle these hygroscopic effects: they are considered globally in space and are described by a differential equation on the mass fraction of the water vapor in the air. This model is made more precise in [6] by taking into account the fact that these effects are local and time-dependent, and leads to the present study.

The current work is hence a natural continuation of the contributions above, and especially of [8]. We follow the same penalization strategy (though another classical approximation strategy) to account for the time-dependence of the domain when dealing with the Navier-Stokes equations. For the Vlasov equation, we apply existence and stability results of DiPerna-Lions type (see [12]) which the authors of [8] obtain. The approximation procedure requires to prove existence and uniqueness for convection-diffusion equations in a time-dependent 
domain using strong compactness results from [25]. As opposed to [8], we were not able to prove interpolation estimates on the moments of the distribution function and therefore we have to assume that there exists a minimal radius for the particles. This hypothesis consists in considering that all the spherical particles are composed of a dry core with common radius (containing the drug) that is surrounded by water. We prove that if all the particles at initial time have this property, then the evaporation of water is not complete and the radius of any given particle remains greater than that of the dry core. It allows to derive fruitful energy estimates linking all the unknowns and to use the same arguments as in [8].

This article is organized as follows. We begin by presenting the model and the main result, and describing the approximation strategy we implement. In Section 3, we recover existence for the approximated system by a fixed-point method, using the Schauder theorem. In order to invoke the latter, we recall results on the Vlasov and Navier-Stokes equations and prove the existence and uniqueness of the global solution to a convection-diffusion equation with Neumann boundary condition in a time-dependent domain. Finally, the last section consists in passing to the limit in the approximated problem in order to obtain a solution to the initial one.

\section{Presentation of the model}

Let us first present the model we investigate in this paper. It extends the model proposed in [6] to a time-dependent domain. Let $\tau>0$ and $\Omega_{0} \subset \mathbb{R}^{3}$ be an open bounded domain with Lipschitz boundary. The variation of the spatial domain is taken into account by means of a mapping $\mathcal{A} \in \mathscr{C}^{2}\left(\mathbb{R}_{+} \times \mathbb{R}^{3} ; \mathbb{R}^{3}\right)$, $(t, \boldsymbol{x}) \mapsto \mathcal{A}(t, \boldsymbol{x})=\mathcal{A}_{t}(\boldsymbol{x})$ such that, for all $t \geq 0, \mathcal{A}_{t}$ is a $\mathscr{C}^{1}$-diffeomorphism and $\mathcal{A}_{0}=\operatorname{Id}_{\mathbb{R}^{3}}$. For every $0 \leq t \leq \tau$, we set $\Omega_{t}=\mathcal{A}_{t}\left(\Omega_{0}\right)$ the bounded domain at time $t$ and

$$
\widehat{\Omega}_{t}=\bigcup_{0<s<t}\{s\} \times \Omega_{s} .
$$

For $t=\tau$, we simply write $\widehat{\Omega}=\widehat{\Omega}_{\tau}$. Furthermore, let

$$
\widehat{\Gamma}=\bigcup_{0<t<\tau}\{t\} \times \partial \Omega_{t}
$$

and, for all $t \in[0, \tau], \boldsymbol{n}_{t}$ be the outgoing unit normal vector field of $\partial \Omega_{t}$. We also use the Eulerian velocity $\boldsymbol{w}$ associated to the flow $t \mapsto \mathcal{A}_{t}$, defined by

$$
\forall(t, \boldsymbol{x}) \in[0, \tau] \times \mathbb{R}^{3}, \quad \boldsymbol{w}\left(t, \mathcal{A}_{t}(\boldsymbol{x})\right)=\partial_{t} \mathcal{A}(t, \boldsymbol{x}) .
$$

Without loss of generality, we assume that $\operatorname{div}_{\boldsymbol{x}} \boldsymbol{w}=0$, so that the Jacobian of the transformation $\mathcal{A}_{t}$ does not depend on $t$ and is therefore constant equal to 1 . Finally, we need to consider the phase space and its boundaries. For any $t \in[0, \tau]$, let us set

$$
\Pi_{t}=\Omega_{t} \times \mathbb{R}^{3} \times \mathbb{R}_{+}^{*} \times \mathbb{R}_{+}^{*}, \quad \widehat{\Pi}_{t}=\widehat{\Omega}_{t} \times \mathbb{R}^{3} \times \mathbb{R}_{+}^{*} \times \mathbb{R}_{+}^{*},
$$




$$
\begin{aligned}
& \Sigma_{t}=\partial \Omega_{t} \times \mathbb{R}^{3} \times \mathbb{R}_{+}^{*} \times \mathbb{R}_{+}^{*}, \quad \widehat{\Sigma}_{t}=\bigcup_{0<s<t}\{s\} \times \partial \Omega_{s} \times \mathbb{R}^{3} \times \mathbb{R}_{+}^{*} \times \mathbb{R}_{+}^{*}, \\
& \widehat{\Pi}=\widehat{\Omega} \times \mathbb{R}^{3} \times \mathbb{R}_{+}^{*} \times \mathbb{R}_{+}^{*}, \quad \widehat{\Sigma}=\widehat{\Gamma} \times \mathbb{R}^{3} \times \mathbb{R}_{+}^{*} \times \mathbb{R}_{+}^{*} .
\end{aligned}
$$

and

$$
\begin{gathered}
\widehat{\Sigma}^{ \pm}=\left\{(t, \boldsymbol{x}, \boldsymbol{v}, r, T) \in \widehat{\Sigma}, \pm(\boldsymbol{v}-\boldsymbol{w}(t, \boldsymbol{x})) \cdot \boldsymbol{n}_{t}(\boldsymbol{x})>0\right\}, \\
\widehat{\Sigma}^{0}=\left\{(t, \boldsymbol{x}, \boldsymbol{v}, r, T) \in \widehat{\Sigma},(\boldsymbol{v}-\boldsymbol{w}(t, \boldsymbol{x})) \cdot \boldsymbol{n}_{t}=0\right\},
\end{gathered}
$$

The particles are composed of an active substance and water. More precisely, we suppose that the active substance lies in a ball the radius of which, denoted by $r_{\text {drug }}>0$, does not change. We assume that this ball is surrounded by water and the whole creates a ball of radius $r$. In particular, all the particles have a radius greater than $r_{\mathrm{drug}}$. If we denote by, respectively, $\rho, \rho_{w}$ and $\rho_{\mathrm{drug}}$ the mass densities of a particle, water and drug, the mass conservation at the microscopic level writes

$$
\rho r^{3}=\rho_{w}\left(r^{3}-r_{\text {drug }}^{3}\right)+\rho_{\text {drug }} r_{\text {drug }}^{3} .
$$

In the rest of this work, we choose $\rho_{w}=1$ and $\rho_{\text {drug }}=2$. Consequently, the radius dependent mass of a particle becomes, up to a multiplication by a positive constant,

$$
m(r)=r^{3}+r_{\text {drug }}^{3} .
$$

In addition to the variation of radius, we consider that the temperature of the particles may vary. Therefore, a particle is characterized at time $t \geq 0$ by its position $\boldsymbol{x} \in \Omega_{t}$, its velocity $\boldsymbol{v} \in \mathbb{R}^{3}$, its radius $r>r_{\text {drug }}$ and its temperature $T>0$. Consequently, we describe the aerosol by a density function $f(t, \boldsymbol{x}, \boldsymbol{v}, r, T)$ which solves the Vlasov-like equation

$$
\partial_{t} f+\boldsymbol{v} \cdot \nabla_{\boldsymbol{x}} f+\operatorname{div}_{\boldsymbol{v}}(\boldsymbol{A} f)+\partial_{r}(a f)+\partial_{T}(b f)=0 \text { in } \widehat{\Pi},
$$

where $\boldsymbol{A}$ is the drag acceleration exerted by the air on the particles, $a$ describes the variation of the radii of the particles and $b$ describes the variation of their temperatures. We prescribe the following absorption condition:

$$
f=0 \quad \text { on } \widehat{\Sigma}^{-},
$$

which means that all the particles reaching the physical boundary are deposited.

We classically assume the air to be a viscous Newtonian incompressible fluid [7. It can therefore be described by its pressure $p(t, \boldsymbol{x})$ and velocity $\boldsymbol{u}(t, \boldsymbol{x})$, which satisfy the incompressible Navier-Stokes equations, with constant mass density and viscosity both taken equal to 1 ,

$$
\begin{gathered}
\partial_{t} \boldsymbol{u}+\left(\boldsymbol{u} \cdot \nabla_{\boldsymbol{x}}\right) \boldsymbol{u}+\nabla_{\boldsymbol{x}} p-\Delta_{\boldsymbol{x}} \boldsymbol{u}=\boldsymbol{F} \quad \text { in } \widehat{\Omega}, \\
\operatorname{div}_{\boldsymbol{x}} \boldsymbol{u}=0 \quad \text { in } \widehat{\Omega},
\end{gathered}
$$

where $\boldsymbol{F}$ is the force applied by the aerosol on the fluid. We prescribe the Dirichlet boundary condition:

$$
\boldsymbol{u}=\boldsymbol{w} \quad \text { on } \widehat{\Gamma} \text {. }
$$


The coupling terms $\boldsymbol{A}$ and $\boldsymbol{F}$ are given by the Stokes law [7] as

$$
\boldsymbol{A}(t, \boldsymbol{x}, \boldsymbol{v}, r)=\frac{r}{r^{3}+r_{\mathrm{drug}}^{3}}(\boldsymbol{u}(t, \boldsymbol{x})-\boldsymbol{v})
$$

and

$$
\boldsymbol{F}(t, \boldsymbol{x})=-\int_{\mathbb{R}^{3} \times \mathbb{R}_{+}^{*} \times \mathbb{R}_{+}^{*}} r(\boldsymbol{u}(t, \boldsymbol{x})-\boldsymbol{v}) f(t, \boldsymbol{x}, \boldsymbol{v}, r, T) .
$$

The variation of the radius of a particle stems from the evaporation of the water it contains or the condensation of the water vapor from the surrounding air. If the water vapor mass fraction at the surface of the particle $Y_{v \text {,surf }}(r, T)$ is smaller than the water vapor mass fraction in the air $Y(t, \boldsymbol{x})$, then a part of the surrounding vapor condensates and the radius of the particle increases. In the opposite case, a part of the water contained in the particle evaporates and the radius decreases. More precisely, [6, formulas (6)-(13)] provide the following expression for the function $a$, when all physical parameters are normalized:

$$
a(t, \boldsymbol{x}, r, T)=\frac{Y(t, \boldsymbol{x})-Y_{v, \text { surf }}(r, T)}{r} .
$$

Note that, given the expression for $Y_{v \text {,surf }}$ in [6], we have $Y_{v \text {,surf }}\left(r_{\text {drug }}, \cdot\right)=0$.

The evaporation and condensation of water also give rise to heat fluxes between the air and the particles, which result in temperature variation. There is also a convective heat flux. Again, [ 6] gives the following expression for $b$ :

$$
b(t, \boldsymbol{x}, r, T)=\frac{Y(t, \boldsymbol{x})-Y_{v, \text { surf }}(r, T)}{r^{2}}+\frac{\Theta(t, \boldsymbol{x})-T}{r^{2}},
$$

where $\Theta$ is the air temperature.

The water vapor in the air is subject to transport phenomena and diffusion and also interacts with the particles. Therefore, we assume that $Y$ solves the following convection-diffusion equation in $\widehat{\Omega}$

$$
\partial_{t} Y+\boldsymbol{u} \cdot \nabla_{\boldsymbol{x}} Y-\operatorname{div}_{\boldsymbol{x}}\left(D_{v}(\Theta) \nabla_{\boldsymbol{x}} Y\right)=-\int_{\mathbb{R}^{3} \times \mathbb{R}_{+}^{*} \times \mathbb{R}_{+}^{*}} r^{2} a f,
$$

where $D_{v}(\Theta)$ is the diffusion coefficient of water vapor in the air at temperature $\Theta$. In fact, we can assume $D_{v} \equiv 1$. Indeed, the following analysis remains true as long as $D_{v} \in L^{\infty}\left(\mathbb{R}_{+}\right)$and $D_{v}$ is bounded from below by a positive constant, and those assumptions are physically relevant in the temperature range for which the model is expected to hold. Note that in [6], the numerical simulations lead to a variation of $D_{v}$ of only $2 \%$. We prescribe the Neumann boundary condition.

$$
\nabla_{\boldsymbol{x}} Y \cdot \boldsymbol{n}_{t}=0 \text { on } \widehat{\Gamma} \text {. }
$$

The variation of the air temperature is also described by a convectiondiffusion equation in $\widehat{\Omega}$ :

$$
\partial_{t} \Theta+\boldsymbol{u} \cdot \nabla_{\boldsymbol{x}} \Theta-\Delta_{\boldsymbol{x}} \Theta=\int_{\mathbb{R}^{3} \times \mathbb{R}_{+}^{*} \times \mathbb{R}_{+}^{*}} r(T-\Theta) f,
$$


with the Neumann boundary condition:

$$
\nabla_{\boldsymbol{x}} \Theta \cdot \boldsymbol{n}_{t}=0 \text { on } \widehat{\Gamma} .
$$

To summarize, the system under study in this article is the following one:

$$
\begin{aligned}
& \partial_{t} f+\boldsymbol{v} \cdot \nabla_{\boldsymbol{x}} f+\operatorname{div}_{\boldsymbol{v}}\left(\frac{r}{r^{3}+r_{\mathrm{drug}}^{3}}(\boldsymbol{u}-\boldsymbol{v}) f\right)+\partial_{r}\left(\frac{Y-Y_{v, \text { surf }}}{r} f\right) \\
& +\partial_{T}\left(\left(\frac{Y-Y_{v, \text { surf }}}{r^{2}}+\frac{\Theta-T}{r^{2}}\right) f\right)=0 \text { in } \widehat{\Pi}, \\
& f=0 \quad \text { on } \widehat{\Sigma}^{-}, \\
& \partial_{t} \boldsymbol{u}+\left(\boldsymbol{u} \cdot \nabla_{\boldsymbol{x}}\right) \boldsymbol{u}+\nabla_{\boldsymbol{x}} p-\Delta_{\boldsymbol{x}} \boldsymbol{u}=-\int_{\mathbb{R}^{3} \times \mathbb{R}_{+}^{*} \times \mathbb{R}_{+}^{*}} r(\boldsymbol{u}-\boldsymbol{v}) f \text { in } \widehat{\Omega}, \\
& \operatorname{div}_{\boldsymbol{x}} \boldsymbol{u}=0 \text { in } \widehat{\Omega}, \\
& \boldsymbol{u}=\boldsymbol{w} \text { on } \widehat{\Gamma}, \\
& \partial_{t} Y+\boldsymbol{u} \cdot \nabla_{\boldsymbol{x}} Y-\Delta_{\boldsymbol{x}} Y+\left(\int_{\mathbb{R}^{3} \times \mathbb{R}_{+}^{*} \times \mathbb{R}_{+}^{*}} r f\right) Y=\int_{\mathbb{R}^{3} \times \mathbb{R}_{+}^{*} \times \mathbb{R}_{+}^{*}} r Y_{v, \text { surf }} f, \\
& \nabla_{\boldsymbol{x}} Y \cdot \boldsymbol{n}_{t}=0 \text { on } \widehat{\Gamma} \text {. } \\
& \partial_{t} \Theta+\boldsymbol{u} \cdot \nabla_{\boldsymbol{x}} \Theta-\Delta_{\boldsymbol{x}} \Theta+\left(\int_{\mathbb{R}^{3} \times \mathbb{R}_{+}^{*} \times \mathbb{R}_{+}^{*}} r f\right) \Theta=\int_{\mathbb{R}^{3} \times \mathbb{R}_{+}^{*} \times \mathbb{R}_{+}^{*}} r T f, \\
& \nabla_{\boldsymbol{x}} \Theta \cdot \boldsymbol{n}_{t}=0 \text { on } \widehat{\Gamma} \text {. }
\end{aligned}
$$

It is supplemented with initial conditions for $f, \boldsymbol{u}, Y$, and $\Theta$ :

$$
\begin{gathered}
f(0, \cdot, \cdot, \cdot)=f^{\text {in }} \quad \text { in } \Pi_{0}, \\
\boldsymbol{u}(0, \cdot)=\boldsymbol{u}^{\text {in }} \quad \text { in } \Omega_{0}, \\
Y(0, \cdot)=Y^{\text {in }} \quad \text { in } \Omega_{0}, \\
\Theta(0, \cdot)=\Theta^{\text {in }} \quad \text { in } \Omega_{0} .
\end{gathered}
$$

Remark 2.1. Note that, since $f$ is nonnegative, solutions $Y$ and $\Theta$ to the previous convection-diffusion equations satisfy, at least formally, the weak maximum principle. Since $0 \leq Y_{v \text {, surf }} \leq 1$, we obtain $0 \leq Y \leq 1$ and $\Theta \geq 0$ for all time $t \in[0, \tau]$ if we assume $0 \leq Y^{\text {in }} \leq 1$ and $\Theta^{\text {in }} \geq 0$. 
Let us now formally compute an energy equality relating the energy dissipation and the exchanges between the air and the particles. Recall the Reynolds formula, for any real-valued function $k: \widehat{\Omega} \rightarrow \mathbb{R}$,

$$
\frac{\mathrm{d}}{\mathrm{d} t} \int_{\Omega_{t}} k=\int_{\Omega_{t}} \partial_{t} k+\int_{\partial \Omega_{t}} k \boldsymbol{w} \cdot \boldsymbol{n}_{t} .
$$

Following the argument in [8, we multiply (4) by $\boldsymbol{u}-\boldsymbol{w}$ and integrate by parts over $\widehat{\Omega}_{t}$ for a fixed value of $t \in[0, \tau]$. Similarly, we multiply 2 by $m(r)|\boldsymbol{v}|^{2} / 2$ and integrate by parts over $\widehat{\Pi}_{t}$. Using $(1,, 5),(6), \sqrt{11}$, and 12 , we obtain

$$
\begin{gathered}
\frac{1}{2} \int_{\Omega_{t}}|\boldsymbol{u}|^{2}+\frac{1}{2} \iint_{\Pi_{t}}\left(r^{3}+r_{\mathrm{drug}}^{3}\right)|\boldsymbol{v}|^{2} f+\iint_{\widehat{\Omega}_{t}}\left|\nabla_{\boldsymbol{x}} \boldsymbol{u}\right|^{2}+\iiint_{\widehat{\Pi}_{t}} r|\boldsymbol{u}-\boldsymbol{v}|^{2} f \\
=\frac{1}{2} \int_{\Omega_{0}}\left|\boldsymbol{u}^{\mathrm{in}}\right|^{2}+\frac{1}{2} \iint_{\Pi_{0}}\left(r^{3}+r_{\mathrm{drug}}^{3}\right)|\boldsymbol{v}|^{2} f^{\mathrm{in}}+\iint_{\Omega_{t}} \boldsymbol{u}(t) \cdot \boldsymbol{w}(t)-\int_{\Omega_{0}} \boldsymbol{u}^{\mathrm{in}} \cdot \boldsymbol{w}(0) \\
-\iint_{\widehat{\Omega}_{t}} \boldsymbol{u} \cdot \partial_{s} \boldsymbol{w}-\iint_{\widehat{\Omega}_{t}}\left(\left(u \cdot \nabla_{\boldsymbol{x}}\right) \boldsymbol{w}\right) \cdot \boldsymbol{u}+\iint_{\widehat{\Omega}_{t}} \nabla_{\boldsymbol{x}} \boldsymbol{u}: \nabla_{\boldsymbol{x}} \boldsymbol{w} \\
+\iiint_{\widehat{\Pi}_{t}} r \boldsymbol{w} \cdot(\boldsymbol{u}-\boldsymbol{v}) f+\frac{3}{2} \iiint_{\widehat{\Pi}_{t}} r\left(Y-Y_{v \text {,surf }}\right) f \\
+\frac{1}{2} \iiint_{\widehat{\Sigma}_{t}}\left(r^{3}+r_{\mathrm{drug}}^{3}\right)|\boldsymbol{v}|^{2} f(\boldsymbol{w}-\boldsymbol{v}) \cdot \boldsymbol{n}_{t},
\end{gathered}
$$

We assume that the water surrounding the active substance does not entirely evaporate. Therefore, the radii of the particles are bounded from below by $r_{\text {drug }}$. This translates into the following assumption:

$$
f(\cdot, \cdot, \cdot, r, \cdot)=0, \quad \text { a.e } r<r_{\text {drug }} .
$$

Therefore, if $f$ is nonnegative, since $0 \leq Y_{v \text {,surf }}, Y \leq 1$, we have

$$
\begin{aligned}
\iiint_{\widehat{\Pi}_{t}} r \boldsymbol{w} \cdot(\boldsymbol{u}-\boldsymbol{v}) f+\iiint_{\widehat{\Pi}_{t}} r\left(Y-Y_{v, \text { surf }}\right) f \\
\leq \frac{1}{2} \iiint_{\widehat{\Pi}_{t}} r|\boldsymbol{u}-\boldsymbol{v}|^{2} f+\frac{\|\boldsymbol{w}\|_{L^{\infty}(\widehat{\Omega})}+2}{r_{\mathrm{drug}}^{2}} \iiint_{\widehat{\Pi}_{t}} r^{3} f .
\end{aligned}
$$

But, if we multiply (2) by $r^{3}$ and integrate over $\widehat{\Pi}_{t}$, thanks to the absorption condition (3) and the Grönwall lemma, we obtain

$$
\iint_{\Pi_{t}} r^{3} f \lesssim 1
$$

where the notation $\lesssim$ indicates that the inequality holds up to a multiplicative constant which can depend on $\tau, \boldsymbol{w}, r_{\text {drug }}$ and the initial data. Using again 
the absorption condition (3), we deduce from the previous estimations and the Grönwall lemma again that

$$
\frac{1}{2} \int_{\Omega_{t}}|\boldsymbol{u}|^{2}+\iint_{\Pi_{t}}\left(r^{3}+r_{\text {drug }}^{3}\right)|\boldsymbol{v}|^{2} f+\iint_{\widehat{\Omega}_{t}}\left|\nabla_{\boldsymbol{x}} \boldsymbol{u}\right|^{2}+\iiint_{\widehat{\Pi}_{t}} r|\boldsymbol{u}-\boldsymbol{v}|^{2} f \lesssim 1 .
$$

Let us now derive an estimate relating the air temperature and the particle distribution. We multiply $(9)$ by $\Theta$ and integrate by parts over $\widehat{\Omega}_{t}$, for fixed $t \in(0, \tau)$. Similarly, we multiply 22 by $r^{3} T^{2} / 2$ and integrate by parts over $\widehat{\Pi}_{t}$. Using (5), (6), 10), (11), and (14), we get

$$
\begin{aligned}
\frac{1}{2} \int_{\Omega_{t}}|\Theta|^{2}+\frac{1}{2} \iint_{\Pi_{t}} r^{3} T^{2} f+\iint_{\widehat{\Omega}_{t}}\left|\nabla_{x} \Theta\right|^{2}+\iiint_{\widehat{\Pi}_{t}} r|\Theta-T|^{2} f \\
\quad \leq 3 \iiint_{\widehat{\Pi}_{t}} r T^{2} f+\iiint_{\widehat{\Pi}_{t}} r T f+\int_{\Omega_{0}}\left|\Theta^{\mathrm{in}}\right|^{2}+\iint_{\Pi_{0}} r^{3} T^{2} f^{\text {in }} .
\end{aligned}
$$

Thanks to (15), the Cauchy-Schwarz inequality, and(16), the Grönwall lemma yields

$$
\frac{1}{2} \int_{\Omega_{t}}|\Theta|^{2}+\frac{1}{2} \iint_{\Pi_{t}} r^{3} T^{2} f+\iint_{\widehat{\Omega}_{t}}\left|\nabla_{\boldsymbol{x}} \Theta\right|^{2}+\iiint_{\widehat{\Pi}_{t}} r|\Theta-T|^{2} f \lesssim 1 .
$$

The previous estimates and the boundary conditions motivate the introduction of the following functional spaces. For $p, q \in[1,+\infty]$ and $m \in \mathbb{N}$, denote

$$
L^{p}\left(0, \tau ; W^{m, q}\left(\Omega_{t}\right)\right)=\left\{\zeta \text { measurable, }\|\zeta\|_{L^{p}\left(0, \tau ; W^{m, q}\left(\Omega_{t}\right)\right)}<\infty\right\}
$$

where we set

$$
\|\zeta\|_{L^{p}\left(0, \tau ; W^{m, q}\left(\Omega_{t}\right)\right)}=\|t \mapsto\| \zeta(t)\left\|_{W^{m, q}\left(\Omega_{t}\right)}\right\|_{L^{p}(0, \tau)},
$$

and

$$
\begin{aligned}
\mathcal{V}_{0} & =\left\{\boldsymbol{\varphi} \in L^{2}\left(0, \tau ; H^{1}\left(\Omega_{t}\right)\right), \operatorname{div}_{\boldsymbol{x}} \varphi=0, \boldsymbol{\varphi}=0 \text { on } \widehat{\Gamma}\right\} \\
\mathscr{V} & =\left\{\boldsymbol{\varphi} \in \mathscr{C}^{1}(\widehat{\widehat{\Omega}}), \operatorname{div}_{\boldsymbol{x}} \varphi=0 \text { in } \widehat{\Omega}, \boldsymbol{\varphi}=0 \text { on } \widehat{\Gamma}, \boldsymbol{\varphi}(\tau)=0\right\} \\
\mathscr{X} & =\left\{\zeta \in \mathscr{C}^{1}(\widehat{\widehat{\Omega}}), \zeta=0 \text { on } \widehat{\Gamma}, \zeta(\tau)=0\right\}
\end{aligned}
$$

and, denoting by $\bar{g}$ the extension on $[0, \tau] \times \mathbb{R}^{3}$ by zero of a function $g$ defined on $\widehat{\Omega}$, let

$$
\begin{gathered}
L^{\infty}\left(0, \tau ; L^{p}\left(\Pi_{t}\right)\right)=\left\{f \text { measurable, } \bar{f} \in L^{\infty}\left(0, \tau ; L^{p}\left(\mathbb{R}^{3} \times \mathbb{R}^{3} \times \mathbb{R}_{+}^{*} \times \mathbb{R}_{+}^{*}\right)\right)\right\}, \\
\mathscr{W}=\left\{\psi \in \mathscr{C}_{c}^{1}\left(\overline{\widehat{\Omega}} \times \mathbb{R}^{3} \times \mathbb{R}_{+}^{*} \times \mathbb{R}_{+}^{*}\right), \psi=0 \text { on } \widehat{\Sigma}^{+} \cup \widehat{\Sigma}^{0}, \psi(\tau)=0\right\} .
\end{gathered}
$$

Let us now state the assumptions on the initial data.

Assumption 1. $Y^{\mathrm{in}} \in L^{2}\left(\Omega_{0}\right)$ and $0 \leq Y^{\mathrm{in}} \leq 1$ in $\Omega_{0}$. 
Assumption 2. $\boldsymbol{u}^{\text {in }} \in L^{2}\left(\Omega_{0}\right)$ and $\operatorname{div}_{\boldsymbol{x}} \boldsymbol{u}^{\text {in }}=0$.

Assumption 3. $\Theta^{\text {in }} \in L^{2}\left(\Omega_{0}\right)$ and $\Theta^{\text {in }}>0$ in $\Omega_{0}$.

Assumption 4. $f^{\text {in }} \in L^{\infty}\left(\Pi_{0}\right)$ is nonnegative, and for almost every $r \leq r_{\mathrm{drug}}$, $f^{\text {in }}(\cdot, \cdot, r, \cdot)=0$, and

$$
\left(1+r^{3}+r^{3}|\boldsymbol{v}|^{2}+\left|Y^{\text {in }}-Y_{v, \text { surf }}\right|\left(\frac{1}{r}+\frac{1}{r^{2}}\right)+\frac{\left|\Theta^{\text {in }}-T\right|}{r^{2}}\right) f^{\text {in }} \in L^{1}\left(\Pi_{0}\right) .
$$

Let us now define the notion of weak solution of the problem.

Definition 2.2. We say that a 4-tuple $(\boldsymbol{u}, Y, \Theta, f)$ is a weak solution of the system (4)-(10) with initial data $\left(\boldsymbol{u}^{\text {in }}, Y^{\text {in }}, \Theta^{\text {in }}, f^{\text {in }}\right)$ if the following conditions are satisfied. The distribution function must verify:

- $\bar{f} \in L^{\infty}\left((0, \tau) \times \mathbb{R}^{3} \times \mathbb{R}^{3} \times \mathbb{R}_{+}^{*} \times \mathbb{R}_{+}^{*}\right) \cap \mathscr{C}^{0}\left([0, \tau] ; L^{p}\left(\mathbb{R}^{3} \times \mathbb{R}^{3} \times \mathbb{R}_{+}^{*} \times \mathbb{R}_{+}^{*}\right)\right)$ for all $p \in[1, \infty)$,

- $\left(1+r^{3}+r^{3}|\boldsymbol{v}|^{2}\right) f \in L^{\infty}\left(0, \tau ; L^{1}\left(\Pi_{t}\right)\right)$,

- $\left(Y-Y_{v, \text { surf }}\right)\left(\frac{1}{r}+\frac{1}{r^{2}}\right) f \in L_{l o c}^{1}(\widehat{\Pi})$,

- $\frac{\Theta-T}{r^{2}} f \in L_{l o c}^{1}(\widehat{\Pi})$.

The fluid quantities must satisfy:

- $\boldsymbol{u} \in L^{\infty}\left(0, \tau ; L^{2}\left(\Omega_{t}\right)\right) \cap L^{2}\left(0, \tau ; H^{1}\left(\Omega_{t}\right)\right)$,

- $\boldsymbol{u}-\boldsymbol{w} \in \mathcal{V}_{0}$,

- $Y \in L^{\infty}\left(0, \tau ; L^{2}\left(\Omega_{t}\right)\right) \cap L^{2}\left(0, \tau ; H^{1}\left(\Omega_{t}\right)\right)$,

- $\Theta \in L^{\infty}\left(0, \tau ; L^{2}\left(\Omega_{t}\right)\right) \cap L^{2}\left(0, \tau ; H^{1}\left(\Omega_{t}\right)\right)$.

Finally, the following weak formulations must hold for any $\psi \in \mathscr{W}, \varphi \in \mathscr{V}$, and $\zeta \in \mathscr{X}:$

$$
\begin{aligned}
\iiint_{\widehat{\Pi}} f\left(\partial_{t} \psi+\boldsymbol{v} \cdot \nabla_{\boldsymbol{x}} \psi+\frac{\boldsymbol{u}-\boldsymbol{v}}{r^{2}+\frac{r_{\mathrm{drug}}^{3}}{r}} \cdot \nabla_{\boldsymbol{v}} \psi\right) & \\
+\iiint_{\widehat{\Pi}} f\left(\frac{Y-Y_{v, \text { surf }}}{r}\left(\partial_{r} \psi+\frac{1}{r} \partial_{T} \psi\right)\right. & \left.\frac{\Theta-T}{r^{2}} \partial_{T} \psi\right) \\
& =-\iint_{\Pi_{0}} f^{\text {in }} \psi(0, \cdot),
\end{aligned}
$$




$$
\begin{gathered}
\iint_{\widehat{\Omega}}\left(\boldsymbol{u} \cdot \partial_{t} \boldsymbol{\varphi}+(\boldsymbol{u} \otimes \boldsymbol{u}): \nabla_{\boldsymbol{x}} \boldsymbol{\varphi}-\nabla_{\boldsymbol{x}} \boldsymbol{u}: \nabla_{\boldsymbol{x}} \boldsymbol{\varphi}\right) \\
=-\iint_{\widehat{\Omega}}\left(\int_{\mathbb{R}^{3} \times \mathbb{R}_{+}^{*} \times \mathbb{R}_{+}^{*}} r(\boldsymbol{v}-\boldsymbol{u}) f\right) \cdot \boldsymbol{\varphi}-\int_{\Omega_{0}} \boldsymbol{u}^{\mathrm{in}} \cdot \boldsymbol{\varphi}(0, \cdot), \\
\iint_{\widehat{\Omega}}\left(-Y \partial_{t} \zeta+\zeta \boldsymbol{u} \cdot \nabla_{\boldsymbol{x}} Y+\nabla_{\boldsymbol{x}} Y \cdot \nabla_{\boldsymbol{x}} \zeta\right) \\
=\iint_{\widehat{\Omega}}\left(\int_{\mathbb{R}^{3} \times \mathbb{R}_{+}^{*} \times \mathbb{R}_{+}^{*}} r\left(Y_{v, \text { surf }}-Y\right) f\right) \zeta+\int_{\Omega_{0}} Y^{\text {in }} \zeta(0, \cdot), \\
\iint_{\widehat{\Omega}}\left(-\Theta \partial_{t} \zeta+\zeta \boldsymbol{u} \cdot \nabla_{\boldsymbol{x}} \Theta+\nabla_{\boldsymbol{x}} \Theta \cdot \nabla_{\boldsymbol{x}} \zeta\right) \\
=\iint_{\widehat{\Omega}}\left(\int_{\mathbb{R}^{3} \times \mathbb{R}_{+}^{*} \times \mathbb{R}_{+}^{*}} r(T-\Theta) f\right) \zeta+\int_{\Omega_{0}} \Theta^{\mathrm{in}} \zeta(0, \cdot) .
\end{gathered}
$$

The main result of this paper can now be properly stated.

Theorem 2.3. Under Assumptions 2] 4, there exists a weak solution to Problem (4)-10) in the sense of Definition 2.2

We follow the same strategy as in [8]. Namely, we solve an approximated system by a fixed-point procedure and recover a solution to the initial problem through compactness properties.

In order to obtain similar estimates as the ones we formally derived above, we need to ensure that $f$ vanishes for radii below some positive value. Therefore, we introduce a function $\eta \in \mathscr{C}^{\infty}\left(\mathbb{R}_{+}\right)$such that $\eta \equiv 0$ on $\left[0, r_{\mathrm{drug}} / 2\right]$ and $\eta \equiv 1$ on $\left[r_{\text {drug }},+\infty\right)$, with $\eta^{\prime} \geq 0$.

To solve the Navier-Stokes equations in a cylindrical domain, we follow a penalization procedure. We then use the Friedrichs approximation to solve the equation. Let $\pi_{L}: L^{2}\left(\mathbb{R}^{3}\right) \rightarrow\left\{\boldsymbol{u} \in L^{2}\left(\mathbb{R}^{3}\right), \operatorname{div}_{\boldsymbol{x}} \boldsymbol{u}=0\right\}$ be the Leray projection. We choose $N \in \mathbb{N}$ and let $P_{N}$ be the orthogonal projection

$$
P_{N}: L^{2}\left(\mathbb{R}^{3}\right) \rightarrow \mathscr{F}_{N}=\left\{\boldsymbol{u} \in L^{2}\left(\mathbb{R}^{3}\right)^{3}, \widehat{\boldsymbol{u}}(\xi)=0, \forall|\boldsymbol{\xi}| \geq N\right\},
$$

where $\widehat{\boldsymbol{u}}$ denotes the Fourier transform of $\boldsymbol{u}$. We also need to truncate the right-hand side of (4), as in [8, to apply standard results for the Friedrichs approximation. To preserve the energy estimate $(17)$, we perform the same truncation in 2 . Let $\chi \in \mathscr{C}^{\infty}(\mathbb{R})$ be an odd, increasing, bounded function, with $0 \leq \chi(v) \leq v$ for all $v \geq 0$. We write $\chi(\boldsymbol{v})=\left(\chi\left(v_{1}\right), \chi\left(v_{2}\right), \chi\left(v_{3}\right)\right)$ for any $\boldsymbol{v} \in \mathbb{R}^{3}$. Furthermore, we also need to truncate the variation of temperature that appears in 21 in order to solve this equation.

Unfortunately, the penalization strategy used for solving the Navier-Stokes equation does not preserve the boundary condition $\boldsymbol{u}=\boldsymbol{w}$. Therefore, we have 
to modify the velocity field for the convection-diffusion equations solved by $Y$ and $\Theta$. For $t \in[0, \tau]$, define the projection

$$
\pi_{\Omega_{t}}: L^{2}\left(\Omega_{t}\right) \rightarrow\left\{\boldsymbol{u} \in H^{1}\left(\Omega_{t}\right), \operatorname{div}_{\boldsymbol{x}} \boldsymbol{u}=0, \boldsymbol{u}_{\mid \partial \Omega_{t}}=0\right\} .
$$

We also need the sets

$$
K_{N}=\{(t, \boldsymbol{x}) \in \widehat{\Omega}, d((t, \boldsymbol{x}), \widehat{\Gamma})<1 / N\},
$$

where $d(\cdot, \widehat{\Gamma})$ denotes the Euclidean distance to the boundary $\widehat{\Gamma}$, defined on $\mathbb{R}_{+} \times \mathbb{R}^{3}$.

We can then consider the following approximated problem:

$$
\partial_{t} f+\boldsymbol{v} \cdot \nabla_{\boldsymbol{x}} f+\operatorname{div}_{\boldsymbol{v}}\left(\boldsymbol{G}_{\mathbf{1}} f\right)+\partial_{r}\left(G_{2} f\right)+\partial_{T}\left(G_{3} f\right)=0 \quad \text { in } \widehat{\Pi},
$$

$$
\begin{aligned}
\partial_{t} \boldsymbol{u}+P_{N} \pi_{\mathrm{L}}\left(\operatorname{div}_{\boldsymbol{x}}(\boldsymbol{u} \otimes \boldsymbol{u})\right)-\Delta_{\boldsymbol{x}} \boldsymbol{u} & \\
+N P_{N} \pi_{\mathrm{L}}\left((\boldsymbol{u}-\boldsymbol{w}) \mathbf{1}_{\widehat{\Omega}^{c}}\right)=P_{N} \pi_{\mathrm{L}} \boldsymbol{F} & \text { in }(0, \tau) \times \mathbb{R}^{3}, \\
\operatorname{div}_{\boldsymbol{x}} \boldsymbol{u}=0 \quad \text { in }(0, \tau) \times \mathbb{R}^{3}, & \\
\partial_{t} Y+\boldsymbol{z}_{N} \cdot \nabla_{\boldsymbol{x}} Y-\Delta_{\boldsymbol{x}} Y+c Y=S_{Y} & \text { in } \widehat{\Omega}, \\
\partial_{t} \Theta+\boldsymbol{z}_{N} \cdot \nabla_{\boldsymbol{x}} \Theta-\Delta_{\boldsymbol{x}} \Theta+c \Theta=S_{T} & \text { in } \widehat{\Omega},
\end{aligned}
$$

where

$$
\begin{gathered}
\boldsymbol{F}=\int_{\mathbb{R}^{3} \times \mathbb{R}_{+}^{*} \times \mathbb{R}_{+}^{*}} \chi(\boldsymbol{v}-\boldsymbol{u}) r \eta \bar{f}, \quad \boldsymbol{z}_{N}=\boldsymbol{w}+\pi_{\Omega_{t}}\left((\boldsymbol{u}-\boldsymbol{w}) \mathbf{1}_{K_{N}}\right), \\
c=\int_{\mathbb{R}^{3} \times \mathbb{R}_{+}^{*} \times \mathbb{R}_{+}^{*}} r \eta f, \quad S_{Y}=\int_{\mathbb{R}^{3} \times \mathbb{R}_{+}^{*} \times \mathbb{R}_{+}^{*}} Y_{v, \text { surf }} r \eta f \\
S_{T}=\int_{\mathbb{R}^{3} \times \mathbb{R}_{+}^{*} \times \mathbb{R}_{+}^{*}} r T \eta f, \quad \boldsymbol{G}_{\mathbf{1}}=\chi(\boldsymbol{u}-\boldsymbol{v}) \frac{\eta}{r^{2}+\frac{r_{\text {drug }}^{3}}{r}}, \\
G_{2}=\frac{\bar{Y}-Y_{v, \text { surf }}}{r} \eta, \quad G_{3}=\frac{1}{r} G_{2}+\frac{\chi(\bar{\Theta}-T)}{r^{2}} \eta .
\end{gathered}
$$

This system is completed with the boundary conditions (3) for $f$, (8) for $Y$, and 100 for $\Theta$. Furthermore, $f, Y, \Theta$ and $\boldsymbol{u}$ satisfy, respectively, the initial conditions 111$),(13), \sqrt{14})$, and $\boldsymbol{u}(0)=P_{N} \overline{\boldsymbol{u}^{\text {in }}}$.

We make the same Assumption 2 on $\boldsymbol{u}^{\text {in }}$ but need stronger assumptions on the other initial data.

Assumption 5. $f^{\text {in }} \in L^{\infty}\left(\Pi_{0}\right)$ is nonnegative and compactly supported and, for almost every $r<r_{\text {drug }}, f^{\text {in }}(\cdot, \cdot, r, \cdot) \equiv 0$, and

$$
\left(1+r^{3}+r^{3}|\boldsymbol{v}|^{2}+\left|Y^{\text {in }}-Y_{v, \text { surf }}\right|\left(\frac{1}{r}+\frac{1}{r^{2}}\right)+\frac{\left|\Theta^{\text {in }}-T\right|}{r^{2}}\right) f^{\text {in }} \in L^{1}\left(\Pi_{0}\right) .
$$


Assumption 6. $Y^{\text {in }} \in H^{1}\left(\Omega_{0}\right)$ and $0 \leq Y^{\text {in }} \leq 1$ in $\Omega_{0}$.

Assumption 7. $\Theta^{\text {in }} \in H^{1}\left(\Omega_{0}\right)$ and $\Theta^{\text {in }} \geq 0$ in $\Omega_{0}$.

Existence of a solution for $(22)-(26)$ with conditions $(3),(8)$ and $(10)$, and initial data satisfying Assumptions 2 and 507 is obtained thanks to the Schauder fixed-point theorem that we recall here in an appropriate setting [4].

Theorem 2.4. Let $E$ be a real normed vector space and $\mathscr{C}$ a closed convex nonempty subset of $E$. If $\Lambda: \mathscr{C} \rightarrow \mathscr{C}$ is a continuous map such that $\Lambda(\mathscr{C})$ is a precompact subset of $E$, then $\Lambda$ has a fixed point.

In the following, we use

$$
\begin{aligned}
E=\{(\boldsymbol{u}, Y, \Theta) \mid & \boldsymbol{u} \in L^{2}\left(0, \tau ; H^{1}\left(\mathbb{R}^{3}\right) \cap \mathscr{C}^{0}\left([0, \tau] ; L^{2}\left(\mathbb{R}^{3}\right)\right),\right. \\
Y \in L^{2}\left(0, \tau ; H^{1}\left(\Omega_{t}\right)\right. & \left.\cap \mathscr{C}^{0}\left([0, \tau] ; L^{2}\left(\Omega_{t}\right)\right)\right) \\
\Theta & \left.\in L^{2}\left(0, \tau ; H^{1}\left(\Omega_{t}\right) \cap \mathscr{C}^{0}\left([0, \tau] ; L^{2}\left(\Omega_{t}\right)\right)\right)\right\}
\end{aligned}
$$

and we first set

$$
\mathscr{C}=\{(\boldsymbol{u}, Y, \Theta) \in E \mid 0 \leq Y \leq 1\},
$$

the real convex to which we apply the theorem being in fact a subset of $\mathscr{C}$, as described below.

The vector-space $E$ is endowed with the following norm. Let $\gamma \in L^{2}\left(\mathbb{R}^{3}\right)$. We define the norm $\|\cdot\|_{E}$ by, for all $(\boldsymbol{u}, Y, \Theta) \in E$,

$$
\|(\boldsymbol{u}, Y, \Theta)\|_{E}=\|Y\|_{L^{2}(\widehat{\Omega})}+\|\Theta\|_{L^{2}(\widehat{\Omega})}+\sup _{t \in[0, \tau]}\|\boldsymbol{u}(t, \cdot) \gamma\|_{L^{1}\left(\mathbb{R}^{3}\right)} .
$$

Note that thanks to $\gamma$, when dealing with the convergence of $\left(\boldsymbol{u}_{n}, Y_{n}, \Theta_{n}\right)$ in $E$, we will only have to prove convergence of $\left(u_{n}\right)_{n \in \mathbb{N}}$ in $L^{\infty}\left(0, \tau ; L^{2}(B)\right)$ for a ball $B$, as long as the sequence is bounded in $L^{\infty}\left(0, \tau ; L^{2}\left(\mathbb{R}^{3}\right)\right)$.

Let us apply Theorem 2.4 to the map $\Lambda: \mathscr{C} \rightarrow \mathscr{C}$, the image of a triplet $(\boldsymbol{u}, Y, \Theta) \in \mathscr{C}$ being the only triplet $(\widetilde{\boldsymbol{u}}, \widetilde{Y}, \widetilde{\Theta})$ satisfying the following systems of equations:

$$
\begin{aligned}
\partial_{t} \widetilde{\boldsymbol{u}}+P_{N} \pi_{\mathrm{L}}\left(\operatorname{div}_{\boldsymbol{x}}(\widetilde{\boldsymbol{u}} \otimes \widetilde{\boldsymbol{u}})\right)-\Delta_{\boldsymbol{x}} \widetilde{\boldsymbol{u}}+N P_{N} \pi_{\mathrm{L}}\left((\widetilde{\boldsymbol{u}}-\boldsymbol{w}) \mathbf{1}_{\widehat{\Omega}^{c}}\right) & =P_{N} \pi_{\mathrm{L}} \boldsymbol{F}_{\boldsymbol{u}, Y, \Theta}, \\
\operatorname{div}_{\boldsymbol{x}} \widetilde{\boldsymbol{u}} & =0 \\
\boldsymbol{u}_{t t=0} & =P_{N} \boldsymbol{u}^{\mathrm{in}}
\end{aligned}
$$

in $(0, \tau) \times \mathbb{R}^{3}$,

$$
\begin{aligned}
\partial_{t} \tilde{Y}+\tilde{\boldsymbol{z}}_{N} \cdot \nabla_{\boldsymbol{x}} \tilde{Y}-\Delta_{\boldsymbol{x}} \tilde{Y}+c_{f_{\boldsymbol{u}, Y, \Theta}} \widetilde{Y} & =S_{Y, f_{\boldsymbol{u}, Y, \Theta}} \\
\nabla_{\boldsymbol{x}} \tilde{Y} \cdot \boldsymbol{n}_{t} & =0 \\
Y_{\mid t=0} & =Y^{\mathrm{in}}
\end{aligned}
$$


and

$$
\begin{aligned}
\partial_{t} \widetilde{\Theta}+\widetilde{\boldsymbol{z}}_{N} \cdot \nabla_{\boldsymbol{x}} \widetilde{\Theta}-\Delta_{\boldsymbol{x}} \widetilde{\Theta}+c_{f_{\boldsymbol{u}, Y, \Theta} \widetilde{\Theta}} & =S_{T, f_{\boldsymbol{u}, Y, \Theta},} \\
\nabla_{\boldsymbol{x}} \widetilde{\Theta} \cdot \boldsymbol{n}_{t} & =0, \\
\Theta_{\mid t=0} & =\Theta^{\text {in }},
\end{aligned}
$$

in $\widehat{\Omega}$, where $f_{\boldsymbol{u}, Y, \Theta}$ is the unique weak solution to the problem

$$
\begin{aligned}
\partial_{t} f+\boldsymbol{v} \cdot \nabla_{\boldsymbol{x}} f+\operatorname{div}_{\boldsymbol{v}}\left(\boldsymbol{G}_{1} f\right)+\partial_{r}\left(G_{2} f\right)+\partial_{T}\left(G_{3} f\right) & =0 \text { on } \widehat{\Pi}, \\
f & =0, \text { on } \widehat{\Sigma}^{-}, \\
f_{\mid t=0} & =f^{\text {in }},
\end{aligned}
$$

with

$$
\begin{gathered}
\boldsymbol{F}_{\boldsymbol{u}, Y, \Theta}=\int_{\mathbb{R}^{3} \times \mathbb{R}_{+}^{*} \times \mathbb{R}_{+}^{*}} \chi(\boldsymbol{v}-\boldsymbol{u}) r \eta \overline{f_{\boldsymbol{u}, Y, \Theta}}, \\
\widetilde{\boldsymbol{z}}_{N}=\boldsymbol{w}-\pi_{\Omega_{t}}\left((\widetilde{\boldsymbol{u}}-\boldsymbol{w}) \mathbf{1}_{K_{N}}\right), \\
c_{f_{\boldsymbol{u}, Y, \Theta}}=\int_{\mathbb{R}^{3} \times \mathbb{R}_{+}^{*} \times \mathbb{R}_{+}^{*}} r \eta \overline{f_{\boldsymbol{u}, Y, \Theta}}, \\
S_{T, f_{\boldsymbol{u}, Y, \Theta}}=\int_{\mathbb{R}^{3} \times \mathbb{R}_{+}^{*} \times \mathbb{R}_{+}^{*}} r T \eta \overline{f_{\boldsymbol{u}, Y, \Theta}}, \\
S_{Y, f_{\boldsymbol{u}, Y, \Theta}}=\int_{\mathbb{R}^{3} \times \mathbb{R}_{+}^{*} \times \mathbb{R}_{+}^{*}} Y_{v, \text { surf }} r \eta \overline{f_{\boldsymbol{u}, Y, \Theta}}, \\
\boldsymbol{G}_{1}=\chi(\boldsymbol{u}-\boldsymbol{v}) \frac{r \eta}{r^{3}+r_{\text {drug }}^{3}}, \quad G_{2}=\frac{\bar{Y}-Y_{v, \text { surf }}}{r} \eta, \quad G_{3}=\frac{1}{r} G_{2}+\frac{\chi(\bar{\Theta}-T)}{r^{2}} \eta .
\end{gathered}
$$

The rest of this article is dedicated to the proof of Theorem 2.3 First, we recall the results obtained in [8] regarding the Vlasov equation in a timedependent domain with boundary condition (37) and its consequences. Then we briefly recall that Problem (27)-29) has a unique strong solution and show that this solution is also the unique solution in a less regular setting. We continue by tackling both convection-diffusion problems $(30)-(32)$ and $(33)-(35)$. Our approach relies on a change of variable in order to work over a fixed domain and we use a standard approximation procedure to prove the existence and uniqueness of a solution to the problem over a fixed domain. We can then apply Theorem 2.4 to $\Lambda$ thanks to compactness results of Aubin-Lions type [25] in a time-dependent framework. Finally, we use compactness again to prove the existence of a solution for a slightly modified version of (2)-(14), where $\eta$ still appears, and the DiPerna-Lions theory to get rid of this truncation. 


\section{Proof of Theorem 2.3}

\subsection{Study of the Vlasov equation}

Let $(\boldsymbol{u}, Y, \Theta) \in \mathscr{C}$. The following result is an immediate consequence of Theorem 3.1, Proposition 3.2 and Remark 3.3 of 8 ].

Theorem 3.1. Problem (36)-(38) has a unique weak solution $f_{\boldsymbol{u}, Y, \Theta} \in L^{\infty}(\widehat{\Pi})$ in the sense of Definition 2.2. Moreover, $\overline{f_{\boldsymbol{u}, Y, \Theta}} \in \mathscr{C}^{0}\left([0, \tau] ; L_{l o c}^{p}\left(\mathbb{R}^{3} \times \mathbb{R}^{3} \times \mathbb{R}_{+}^{*} \times\right.\right.$ $\left.\mathbb{R}_{+}^{*}\right)$ ) for all $1 \leq p<\infty$. The trace of $f_{\boldsymbol{u}, Y, \Theta}$ on $\widehat{\Sigma}$ is well-defined as the unique element $\gamma f \in L^{\infty}(\widehat{\Sigma})$ such that, for all test functions $\psi \in \mathscr{D}\left(\mathbb{R} \times \mathbb{R}^{3} \times \mathbb{R}^{3} \times \mathbb{R} \times \mathbb{R}\right)$, all $\beta \in \mathscr{C}^{1}(\mathbb{R})$, and all $0 \leq t_{0} \leq t_{1} \leq \tau$,

$$
\begin{gathered}
\int_{t_{0}}^{t_{1}} \iint_{\Pi_{t}} \beta(f)\left(\partial_{t} \psi+\boldsymbol{v} \cdot \nabla_{\boldsymbol{x}} \psi+\boldsymbol{G}_{\mathbf{1}} \cdot \nabla_{\boldsymbol{v}} \psi+G_{2} \partial_{r} \psi+G_{3} \partial_{T} \psi\right) \\
-\int_{t_{0}}^{t_{1}} \iint_{\Pi_{t}}\left(f \beta^{\prime}(f)-\beta(f)\right) \psi\left(\operatorname{div}_{\boldsymbol{v}} \boldsymbol{G}_{\mathbf{1}}+\partial_{r} G_{2}+\partial_{T} G_{3}\right) \\
=\iint_{\Pi_{t_{1}}} \beta\left(f\left(t_{1}\right)\right) \psi\left(t_{1}\right)-\iint_{\Pi_{t_{0}}} \beta\left(f\left(t_{0}\right)\right) \psi\left(t_{0}\right) \\
+\int_{t_{0}}^{t_{1}} \iint_{\Sigma_{t}} \beta(\gamma f) \psi \boldsymbol{v} \cdot \boldsymbol{n}_{t} .
\end{gathered}
$$

Furthermore, $f$ is nonnegative, so is $\gamma f, f$ is compactly supported, and

$$
\left\|\overline{f_{\boldsymbol{u}, Y, \Theta}}\right\|_{L^{\infty}\left(0, \tau ; L^{p}\left(\mathbb{R}^{3} \times \mathbb{R}^{3} \times \mathbb{R}_{+}^{*} \times \mathbb{R}_{+}^{*}\right)\right)} \lesssim\left\|f^{\text {in }}\right\|_{L^{p}\left(\Pi_{0}\right)} .
$$

We also have $S_{T, f_{u, Y, \Theta}}, S_{Y, f_{u}, Y, \Theta}, c_{f_{u, Y, \Theta}} \in \mathscr{C}^{0}\left([0, \tau] ; L^{p}\left(\Omega_{t}\right)\right)$ for all $p \in[1,+\infty)$ and, for all $p \in[1,+\infty]$,

$$
\begin{aligned}
&\left\|S_{T, f_{\boldsymbol{u}, Y, \Theta}}\right\|_{L^{\infty}\left(0, \tau ; L^{p}\left(\Omega_{t}\right)\right)} \lesssim\left\|f^{\text {in }}\right\|_{L^{p}\left(\Pi_{0}\right)}, \\
&\left\|S_{Y, f_{\boldsymbol{u}, Y, \Theta}}\right\|_{L^{\infty}\left(0, \tau ; L^{p}\left(\Omega_{t}\right)\right)} \lesssim\left\|f^{\text {in }}\right\|_{L^{p}\left(\Pi_{0}\right)},
\end{aligned}
$$

and

$$
\left\|c_{f_{u, Y, \Theta}}\right\|_{L^{\infty}\left(0, \tau ; L^{p}\left(\Omega_{t}\right)\right)} \lesssim\left\|f^{\mathrm{in}}\right\|_{L^{p}\left(\Pi_{0}\right)} .
$$

Finally, $\boldsymbol{F}_{\boldsymbol{u}, Y, \Theta} \in \mathscr{C}^{0}\left([0, \tau] ; L^{2}\left(\mathbb{R}^{3}\right)\right)$ and

$$
\left\|\boldsymbol{F}_{\boldsymbol{u}, Y, \Theta}\right\|_{L^{\infty}\left(0, \tau ; L^{2}\left(\mathbb{R}^{3}\right)\right)} \lesssim\left\|f^{\mathrm{in}}\right\|_{L^{2}\left(\Pi_{0}\right)} .
$$

Remark 3.2. Let us be more accurate with respect to the multiplicative constant appearing in (39). It depends on $p, \tau, r_{\mathrm{drug}},\left\|\eta_{r}^{\prime}\right\|_{L^{\infty}(\mathbb{R})},\left\|\nabla Y_{v, \text { surf }}\right\|_{L^{\infty}\left(\mathbb{R}_{+}^{*} \times \mathbb{R}_{+}^{*}\right)}$ and $\left\|\chi^{\prime}\right\|_{L^{\infty}(\mathbb{R})}$. Therefore, we need to be vigilant when choosing a sequence of truncations $\left(\chi_{n}\right)_{n \in \mathbb{N}}$ in Subsection 3.5 . 
Remark 3.3. The fact that the solution $f$ remains compactly supported comes from the fact that $\boldsymbol{G}_{1}, G_{2}, G_{3} \in L^{\infty}\left((0, \tau) \times \mathbb{R}^{3} \times \mathbb{R}^{3} \times \mathbb{R}_{+}^{*} \times \mathbb{R}_{+}^{*}\right)$. It is a standard result that the size of the support only depends on the initial datum and the $L^{1}\left(0, \tau ; L^{\infty}\left(\mathbb{R}^{3} \times \mathbb{R}^{3} \times \mathbb{R}_{+}^{*} \times \mathbb{R}_{+}^{*}\right)\right)$ norm of $\boldsymbol{G}_{\mathbf{1}}, G_{2}, G_{3}$. The only parameter involved in the bounds (40)-(43) that is of interest is the size of the support of $f$, and therefore the $L^{\infty}$ norms of $\left|\boldsymbol{G}_{\mathbf{1}}\right|+\left|G_{2}\right|+\left|G_{3}\right|$.

\subsection{Study of the approximated Navier-Stokes equations}

Let $\boldsymbol{u}^{\text {in }} \in L^{2}\left(\mathbb{R}^{3}\right)$ such that $\operatorname{div}_{\boldsymbol{x}} \boldsymbol{u}^{\text {in }}=0$ and let $\boldsymbol{F} \in \mathscr{C}^{0}\left([0, \tau] ; L^{2}\left(\mathbb{R}^{3}\right)\right)$. Our focus in this section is the following problem on $(0, \tau) \times \mathbb{R}^{3}$ :

$$
\begin{aligned}
\partial_{t} \boldsymbol{u}+P_{N} \pi_{\mathrm{L}}\left(\operatorname{div}_{\boldsymbol{x}}(\boldsymbol{u} \otimes \boldsymbol{u})\right)-\Delta_{\boldsymbol{x}} \boldsymbol{u}+N P_{N} \pi_{\mathrm{L}}\left((\boldsymbol{u}-\boldsymbol{w}) \mathbf{1}_{\widehat{\Omega}^{c}}\right) & =P_{N} \pi_{\mathrm{L}} \boldsymbol{F}, \\
\operatorname{div}_{\boldsymbol{x}} \boldsymbol{u} & =0, \\
\boldsymbol{u}_{\mid t=0} & =P_{N} \boldsymbol{u}^{\mathrm{in}} .
\end{aligned}
$$

In this section, $\lesssim_{N}$ will denote an inequality up to a multiplicative constant which can depend on $\tau, \boldsymbol{w}$ and $N$. Applying the Cauchy-Lipschitz theorem and the standard procedure to obtain the energy estimates leads to the following result.

Theorem 3.4. Problem (44)- 46 has a unique solution $\boldsymbol{u} \in \mathscr{C}^{1}\left([0, \tau] ; \mathscr{F}_{N}\right)$. Furthermore, for all $t \in[0, \tau]$,

$$
\|\boldsymbol{u}(t)\|_{L^{2}\left(\mathbb{R}^{3}\right)}^{2}+\int_{0}^{t}\left\|\nabla_{\boldsymbol{x}} \boldsymbol{u}\right\|_{L^{2}\left(\mathbb{R}^{3}\right)}^{2} \mathrm{~d} s \lesssim_{N} 1+\left\|\boldsymbol{u}^{\mathrm{in}}\right\|_{L^{2}\left(\mathbb{R}^{3}\right)}^{2}+\|\boldsymbol{F}\|_{L^{2}\left((0, \tau) \times \mathbb{R}^{3}\right)}^{2}
$$

and

$$
\begin{aligned}
&\left\|\partial_{t} \boldsymbol{u}\right\|_{L^{2}\left((0, \tau) \times \mathbb{R}^{3}\right)}^{2}+\left\|\nabla_{\boldsymbol{x}} \boldsymbol{u}\right\|_{L^{\infty}\left(0, \tau ; L^{2}\left(\mathbb{R}^{2}\right)\right)}^{2}+\|\boldsymbol{u}\|_{L^{2}\left(0, \tau ; H^{2}\left(\Omega_{t}\right)\right)}^{2} \\
& \lesssim_{N} 1+\left\|\boldsymbol{u}^{\mathrm{in}}\right\|_{L^{2}\left(\mathbb{R}^{3}\right)}^{2}+\|\boldsymbol{F}\|_{L^{2}\left((0, \tau) \times \mathbb{R}^{3}\right)}^{2} .
\end{aligned}
$$

We also use a uniqueness result for weaker solutions of 44- (46) with respect to the time variable.

Definition 3.5. We say that $\boldsymbol{u}_{*} \in \mathscr{C}\left([0, \tau] ; L_{l o c}^{2}\left(\mathbb{R}^{3}\right)\right) \cap L^{2}\left(0, \tau ; \mathscr{F}_{N}\right)$ is a weakin-time solution of (44)-46) if, for all $\varphi \in \mathscr{D}\left((0, \tau) \times \mathbb{R}^{3}\right)$ such that $\operatorname{div}_{\boldsymbol{x}} \varphi=0$,

$$
\begin{aligned}
\int_{0}^{\tau} \int_{\mathbb{R}^{3}}( & \left.-\boldsymbol{u}_{*} \cdot \partial_{t} \boldsymbol{\varphi}+P_{N} \pi_{L}\left(\operatorname{div}_{\boldsymbol{x}}\left(\boldsymbol{u}_{*} \otimes \boldsymbol{u}_{*}\right)\right) \cdot \varphi+\nabla_{\boldsymbol{x}} \boldsymbol{u}_{*}: \nabla_{\boldsymbol{x}} \boldsymbol{\varphi}\right) \\
& +\int_{0}^{\tau} \int_{\mathbb{R}^{3}} N P_{N} \pi_{L}\left(\left(\boldsymbol{u}_{*}-\boldsymbol{w}\right) \mathbf{1}_{\widehat{\Omega}^{c}}\right) \cdot \boldsymbol{\varphi}=\int_{0}^{\tau} \int_{\mathbb{R}^{3}} P_{N} \pi_{L}(\boldsymbol{F}) \cdot \boldsymbol{\varphi},
\end{aligned}
$$

with $\boldsymbol{u}_{*}(0)=P_{N} \boldsymbol{u}^{\text {in }}$ and $\operatorname{div}_{\boldsymbol{x}} \boldsymbol{u}_{*}=0$.

Theorem 3.6. The solution $\boldsymbol{u} \in \mathscr{C}^{1}\left([0, \tau] ; \mathscr{F}_{N}\right)$ given by Theorem 3.4 is the only weak-in-time solution of (44)-46] in the sense of Definition 3.5.

This results stems from Definition 3.5 and the derivation of a standard energy estimate. 


\subsection{Study of the convection-diffusion equations}

Note that the equations on $Y$ and $\Theta$ display the same structure. Therefore, we consider a more general framework covering both cases. Let $V^{\text {in }} \in H^{1}\left(\Omega_{0}\right)$, $\boldsymbol{z} \in L^{\infty}\left(0, \tau ; H^{1}\left(\Omega_{t}\right)\right)$ such that $\operatorname{div}_{\boldsymbol{x}} \boldsymbol{z}=0$ and $\boldsymbol{z}_{\mid \partial \Omega_{t}}=\boldsymbol{w}_{\mid \partial \Omega_{t}}$ for almost every $t \in(0, \tau)$. Let $c \in L^{\infty}\left(0, \tau ; L^{4}\left(\Omega_{t}\right)\right)$ and $S \in L^{2}(\widehat{\Omega})$ such that $c$ and $S$ are nonnegative.

This section is dedicated to solving the problem

$$
\begin{aligned}
\partial_{t} V+\boldsymbol{z} \cdot \nabla_{\boldsymbol{x}} V-\Delta_{\boldsymbol{x}} V+c V & =S & & \text { in } \widehat{\Omega}, \\
\nabla_{\boldsymbol{x}} V \cdot \boldsymbol{n}_{t} & =0 & & \text { on } \widehat{\Gamma}, \\
V(0, \cdot) & =V^{\text {in }} & & \text { in } \Omega_{0} .
\end{aligned}
$$

The notion of weak solution of this problem is defined as follows.

Definition 3.7. We say that $V \in \mathscr{C}^{0}\left([0, \tau] ; L^{2}\left(\Omega_{t}\right)\right) \cap L^{2}\left(0, \tau ; H^{1}\left(\Omega_{t}\right)\right)$ is a weak solution of (50)-(52) if $V(0)=V^{\text {in }}$ and, for all $\zeta \in \mathscr{C}^{1}(\bar{\Omega})$, for all $t \in(0, \tau)$,

$$
\int_{\Omega_{t}} V(t) \zeta(t)-\int_{\Omega_{0}} V^{\mathrm{in}} \zeta(0)-\iint_{\widehat{\Omega}_{t}} V \partial_{s} \zeta+\int_{0}^{t} B(V(s), \zeta(s) ; s)=\iint_{\widehat{\Omega}_{t}} S \zeta
$$

where $B$ is the time-dependent bilinear form defined, for all $\left(v_{1}, v_{2}\right) \in H^{1}\left(\Omega_{t}\right)^{2}$ and almost every $t \in(0, \tau)$, by

$$
B\left(v_{1}, v_{2} ; t\right)=\int_{\Omega_{t}}\left(\nabla_{\boldsymbol{x}} v_{1} \cdot \nabla_{\boldsymbol{x}} v_{2}+v_{2} \boldsymbol{z}(t) \cdot \nabla_{\boldsymbol{x}} v_{1}+c(t) v_{1} v_{2}\right) .
$$

We will prove the following result.

Theorem 3.8. Problem (50)-(52) has a unique weak solution $V$ in the sense of Definition 3.7. Furthermore, $V$ satisfies the weak maximum principle: if $V^{\text {in }}$ is nonnegative on $\Omega_{0}$, then $V$ is nonnegative on $\widehat{\Omega}$. Moreover, for all $t \in(0, \tau)$,

$$
\|V(t)\|_{L^{2}\left(\Omega_{t}\right)}^{2}+2 \int_{0}^{t}\left\|\nabla_{\boldsymbol{x}} V\right\|_{L^{2}\left(\Omega_{s}\right)}^{2}+2 \iint_{\widehat{\Omega}_{t}} c V \lesssim\left\|V^{\mathrm{in}}\right\|_{L^{2}\left(\Omega_{0}\right)}^{2}+\|S\|_{L^{2}(\widehat{\Omega})}^{2} .
$$

Finally, for all $\zeta \in \mathscr{D}(\widehat{\Omega})$,

$$
\begin{aligned}
\left|\left\langle\partial_{t} V, \zeta\right\rangle\right| & \lesssim\left(\left\|V^{\mathrm{in}}\right\|_{L^{2}\left(\Omega_{0}\right)}^{2}+\|S\|_{L^{2}\left(0, \tau ; L^{2}\left(\Omega_{t}\right)\right)}^{2}\right) \\
& \times\left(1+\|\boldsymbol{z}\|_{L^{\infty}\left(0, \tau ; L^{2}\left(\Omega_{t}\right)\right)}^{2}+\|c\|_{L^{\infty}\left(0, \tau ; L^{4}\left(\Omega_{t}\right)\right)}^{2}\right)\|\zeta\|_{L^{2}\left(0, \tau ; H^{2}\left(\Omega_{t}\right)\right)},
\end{aligned}
$$

where $\langle\cdot, \cdot\rangle$ denotes the duality bracket $\mathscr{D}^{\prime}(\widehat{\Omega})-\mathscr{D}(\widehat{\Omega})$.

In order to prove this theorem, we perform a change of variable so as to work in a fixed domain. For $t \in(0, \tau)$ and $\boldsymbol{y} \in \Omega_{0}$, let

$$
V^{0}(t, \boldsymbol{y})=V\left(t, \mathcal{A}_{t}(\boldsymbol{y})\right), \quad \boldsymbol{z}^{0}(t, \boldsymbol{y})=\boldsymbol{z}\left(t, \mathcal{A}_{t}(\boldsymbol{y})\right),
$$




$$
\begin{gathered}
\boldsymbol{w}^{0}(t, \boldsymbol{y})=\boldsymbol{w}\left(t, \mathcal{A}_{t}(\boldsymbol{y})\right), \quad c^{0}(t, \boldsymbol{y})=c\left(t, \mathcal{A}_{t}(\boldsymbol{y})\right), \\
S^{0}(t, \boldsymbol{y})=S\left(t, \mathcal{A}_{t}(\boldsymbol{y})\right), \quad J(t, \boldsymbol{y})=\operatorname{Cof}\left(\nabla_{\boldsymbol{y}} \mathcal{A}_{t}(\boldsymbol{y})\right), \quad D(t, \boldsymbol{y})=J(t, \boldsymbol{y})^{\top} J(t, \boldsymbol{y}),
\end{gathered}
$$

and consider the problem on $(0, \tau) \times \Omega_{0}$

$$
\begin{aligned}
\partial_{t} V^{0}+\left(\boldsymbol{z}^{0}-\boldsymbol{w}^{0}\right) \cdot J \nabla_{\boldsymbol{y}} V^{0}-\operatorname{div}_{\boldsymbol{y}}\left(D \nabla_{\boldsymbol{y}} V^{0}\right)+c^{0} V^{0} & =S^{0}, \\
D \nabla_{\boldsymbol{y}} V^{0} \cdot \boldsymbol{n}_{0} & =0, \\
V^{0}(0, \cdot) & =V^{\mathrm{in}} .
\end{aligned}
$$

Definition 3.9. We say that $V^{0} \in \mathscr{C}^{0}\left([0, \tau] ; L^{2}\left(\Omega_{0}\right)\right) \cap L^{2}\left(0, \tau ; H^{1}\left(\Omega_{0}\right)\right)$ such that $\partial_{t} V^{0} \in L^{2}\left(0, \tau ; H^{1}\left(\Omega_{0}\right)^{\prime}\right)$ is a weak solution to Problem (56)-(58) if $V^{0}(0)=$ $V^{\text {in }}$ and, for all $\zeta \in \mathscr{C}^{1}\left(\overline{\Omega_{0}}\right)$, for any $t \in(0, \tau)$,

$$
\int_{0}^{t}\left\langle\partial_{t} V^{0}, \zeta\right\rangle_{1}+\int_{0}^{t} \int_{\Omega_{0}} B^{0}\left(V^{0}(s), \zeta(s) ; s\right)=\int_{0}^{t} \int_{\Omega_{0}} S^{0} \zeta
$$

where $\langle\cdot, \cdot\rangle_{1}$ denotes the duality bracket $H^{1}\left(\Omega_{0}\right)^{\prime}-H^{1}\left(\Omega_{0}\right)$ and $B^{0}$ is the timedependent bilinear form defined, for all $\left(v_{1}, v_{2}\right) \in H^{1}\left(\Omega_{0}\right)^{2}$ and almost every $t \in(0, \tau)$, by

$$
B^{0}\left(v_{1}, v_{2} ; t\right)=\int_{\Omega_{0}}\left[D(t) \nabla_{\boldsymbol{y}} v_{1} \cdot \nabla_{\boldsymbol{y}} v_{2}+v_{2}\left(\boldsymbol{z}^{0}-\boldsymbol{w}^{0}\right)(t) \cdot J(t) \nabla_{\boldsymbol{y}} v_{1}+c^{0}(t) v_{1} v_{2}\right] .
$$

Note that $\operatorname{div}_{\boldsymbol{y}} J=0$ and that $\operatorname{div}_{\boldsymbol{x}} \boldsymbol{w}=0$ implies that $\operatorname{det} J=1$ as well as $\operatorname{div}_{\boldsymbol{y}}\left(J^{\top} \boldsymbol{w}^{0}\right)=0$. Therefore, by a change of variable, one can easily check that $V$ is a weak solution of Problem (50)-(52) in the sense of Definition 3.7 if and only if $V^{0}$ is a weak solution of Problem (56)-(58) in the sense of Definition 3.9.

Let us now focus on Problem (56)-(58). In order to prove the existence of a weak solution, we follow the steps of [14], which deals with the Dirichlet boundary condition rather than the Neumann one, and use a Galerkin approximation. Let $\left(v_{n}\right)_{n \in \mathbb{N}}$ be an orthogonal basis of $H^{1}\left(\Omega_{0}\right)$ such that $\left(v_{n}\right)_{n \in \mathbb{N}}$ is an orthonormal basis of $L^{2}\left(\Omega_{0}\right)$ and, for all $n \in \mathbb{N}$, let $\mathcal{H}_{n}=\operatorname{Span}\left(v_{0}, \ldots, v_{n}\right)$. Let $n \in \mathbb{N}$. By the linear Cauchy-Lipschitz theorem, there exists a unique solution $V_{n}^{0} \in W^{1, \infty}\left(0, \tau ; \mathcal{H}_{n}\right)$ to the problem

$$
\begin{aligned}
\int_{\Omega_{0}} \partial_{t} V_{n}^{0} v_{k}+B^{0}\left(V_{n}^{0}(t), v_{k} ; t\right) & =\int_{\Omega_{0}} S^{0} v_{k} \quad \text { a.e. } t \in(0, \tau), \forall k \in \llbracket 0, n \rrbracket \\
\left.V_{n}^{0}\right|_{t=0} & =P_{\mathcal{H}_{n}} V^{\text {in }} .
\end{aligned}
$$

We write

$$
V_{n}^{0}(t, \boldsymbol{y})=\sum_{k=0}^{n} q_{k}^{n}(t) v_{k}(\boldsymbol{y}) .
$$

Then, if we multiply 60 by $q_{k}^{n}$, sum over $k$ for each $k$ and integrate on $(0, t)$, 
for $t \in(0, \tau)$, we get

$$
\begin{array}{r}
\int_{0}^{t} \int_{\Omega_{0}}\left(V_{n}^{0} \partial_{s} V_{n}^{0}+V_{n}^{0}\left(\boldsymbol{z}^{0}-\boldsymbol{w}^{0}\right) \cdot J \nabla_{\boldsymbol{y}} V_{n}^{0}+D \nabla_{\boldsymbol{y}} V_{n}^{0} \cdot \nabla_{\boldsymbol{y}} V_{n}^{0}+c^{0}\left|V_{n}^{0}\right|^{2}\right) \\
=\int_{0}^{t} \int_{\Omega_{0}} S^{0} V_{n}^{0} .
\end{array}
$$

First note that

$$
\int_{0}^{t} \int_{\Omega_{0}} V_{n}^{0} \partial_{s} V_{n}^{0}=\frac{1}{2}\left\|V_{n}^{0}(t)\right\|_{L^{2}\left(\Omega_{0}\right)}^{2}-\frac{1}{2}\left\|V_{n}^{0}(0)\right\|_{L^{2}\left(\Omega_{0}\right)}^{2} .
$$

Furthermore, for almost every $t \in(0, \tau)$,

$$
\begin{aligned}
\int_{\Omega_{0}} V_{n}^{0}\left(\boldsymbol{z}^{0}-\boldsymbol{w}^{0}\right) \cdot J \nabla_{\boldsymbol{y}} V_{n}^{0}= & \int_{\partial \Omega_{0}}\left|V_{n}^{0}\right|^{2}\left(\boldsymbol{z}^{0}-\boldsymbol{w}^{0}\right) \cdot J \boldsymbol{n}_{0} \\
-\int_{\Omega_{0}} V_{n}^{0}\left(\boldsymbol{z}^{0}-\boldsymbol{w}^{0}\right) \cdot J \nabla_{\boldsymbol{y}} V_{n}^{0} & -\int_{\Omega_{0}}\left|V_{n}^{0}\right|^{2}\left(\operatorname{div}_{\boldsymbol{y}} J\right) \cdot\left(\boldsymbol{z}^{0}-\boldsymbol{w}^{0}\right) \\
& -\int_{\Omega_{0}}\left|V_{n}^{0}\right|^{2} \operatorname{Tr}\left(J^{\top} \nabla_{\boldsymbol{y}}\left(\boldsymbol{z}^{0}-\boldsymbol{w}^{0}\right)\right) .
\end{aligned}
$$

Since $\operatorname{div}_{\boldsymbol{y}} J=0$ and

$$
\begin{array}{r}
\operatorname{Tr}\left(J^{\top} \nabla_{\boldsymbol{y}}\left(\boldsymbol{z}^{0}-\boldsymbol{w}^{0}\right)\right)=\operatorname{Tr}\left[\operatorname{Cof}\left(\nabla_{\boldsymbol{y}} \mathcal{A}_{t}(\boldsymbol{y})\right)^{\top} \nabla_{\boldsymbol{x}}(\boldsymbol{z}-\boldsymbol{w})\left(t, \mathcal{A}_{t}(\boldsymbol{y})\right) \nabla_{\boldsymbol{y}} \mathcal{A}_{t}(\boldsymbol{y})\right] \\
=\frac{1}{\operatorname{det}\left(\nabla_{\boldsymbol{y}} \mathcal{A}_{t}(\boldsymbol{y})\right)} \operatorname{div}_{\boldsymbol{x}}(\boldsymbol{z}-\boldsymbol{w})\left(t, \mathcal{A}_{t}(\boldsymbol{y})\right)=0,
\end{array}
$$

we deduce from 62 that

$$
\int_{\Omega_{0}} V_{n}^{0}\left(\boldsymbol{z}^{0}-\boldsymbol{w}^{0}\right) \cdot J \nabla_{\boldsymbol{y}} V_{n}^{0}=0
$$

Finally, noticing that $(t, \boldsymbol{y}) \mapsto \sigma(J(t, \boldsymbol{y}))$ is a continuous map from $[0, \tau] \times \overline{\Omega_{0}}$ to $\mathbb{R}^{3 \times 3}$, and since $(0, \tau) \times \overline{\Omega_{0}}$ is compact and $\operatorname{det} J=1$, there exists $\alpha>0$ such that

$$
\int_{0}^{t} \int_{\Omega_{0}} D \nabla_{\boldsymbol{y}} V_{n}^{0} \cdot \nabla_{\boldsymbol{y}} V_{n}^{0} \geq \alpha \int_{0}^{t} \int_{\Omega_{0}}\left|\nabla_{\boldsymbol{y}} V_{n}^{0}\right|^{2}
$$

Therefore,

$$
\begin{aligned}
\left\|V_{n}^{0}(t)\right\|_{L^{2}\left(\Omega_{0}\right)}^{2}+2 \alpha & \int_{0}^{t} \int_{\Omega_{0}}\left|\nabla_{\boldsymbol{y}} V_{n}^{0}\right|^{2}+2 \int_{0}^{t} \int_{\Omega_{0}} c^{0}\left|V_{n}^{0}\right|^{2} \\
& \leq\left\|V^{\mathrm{in}}\right\|_{L^{2}\left(\Omega_{0}\right)}^{2}+\left\|S^{0}\right\|_{L^{2}\left((0, \tau) \times \Omega_{0}\right)}^{2}+\int_{0}^{t}\left\|V_{n}^{0}(s)\right\|_{L^{2}\left(\Omega_{0}\right)}^{2}
\end{aligned}
$$

and by Grönwall's lemma,

$$
\left\|V_{n}^{0}(t)\right\|_{L^{2}\left(\Omega_{0}\right)}^{2}+2 \alpha \int_{0}^{t}\left\|\nabla_{\boldsymbol{y}} V_{n}^{0}\right\|_{L^{2}\left(\Omega_{0}\right)}^{2}+2 \int_{0}^{t} \int_{\Omega_{0}} c^{0}\left|V_{n}^{0}\right|^{2} \lesssim 1
$$


where the underlying constant depends on $V^{\text {in }}$ and $S$. Thus, there exists $V^{0} \in$ $L^{\infty}\left(0, \tau ; L^{2}\left(\Omega_{0}\right)\right) \cap L^{2}\left(0, \tau ; H^{1}\left(\Omega_{0}\right)\right)$ such that, up to a subsequence, $V_{n}^{0} \rightarrow V^{0}$ and $\nabla_{\boldsymbol{y}} V_{n}^{0} \rightarrow \nabla_{\boldsymbol{y}} V^{0}$ weakly in $L^{2}\left((0, \tau) \times \Omega_{0}\right)$ and $V_{n}^{0} \rightarrow V^{0}$ weakly-* in $L^{\infty}\left(0, \tau ; L^{2}\left(\Omega_{0}\right)\right)$.

In order to take the limit $n \rightarrow+\infty$ in $60-61)$, we prove that $\left(\partial_{t} V_{n}^{0}\right)_{n \in \mathbb{N}}$ is bounded in $L^{2}\left(0, \tau ; H^{1}\left(\Omega_{0}\right)^{\prime}\right)$. Let $v \in H^{1}\left(\Omega_{0}\right)$. Applying 60 for $n \in \mathbb{N}$ and almost every $t \in(0, \tau)$ yields

$$
\begin{aligned}
&\left|\left\langle\partial_{t} V_{n}^{0}, v\right\rangle_{1}\right|=\left|\int_{\Omega_{0}} \partial_{t} V_{n}^{0} P_{\mathcal{H}_{n}} v\right| \\
& \leq\left|\int_{\Omega_{0}}\left(P_{\mathcal{H}_{n}} v\right) J^{\top}\left(\boldsymbol{z}^{0}-\boldsymbol{w}^{0}\right) \cdot \nabla_{\boldsymbol{y}} V_{n}^{0}\right|+\left|\int_{\Omega_{0}} D \nabla_{\boldsymbol{y}} V_{n}^{0} \cdot \nabla_{\boldsymbol{y}}\left(P_{\mathcal{H}_{n}} v\right)\right| \\
& \quad+\left|\int_{\Omega_{0}} c^{0} V_{n}^{0}\left(P_{\mathcal{H}_{n}} v\right)\right|+\left|\int_{\Omega_{0}} S^{0}\left(P_{\mathcal{H}_{n}} v\right)\right| .
\end{aligned}
$$

Since

$$
\begin{aligned}
\left|\int_{\Omega_{0}}\left(P_{\mathcal{H}_{n}} v\right) J^{\top}\left(\boldsymbol{z}^{0}-\boldsymbol{w}^{0}\right) \cdot \nabla_{\boldsymbol{y}} V_{n}^{0}\right| & \\
& \lesssim\left\|\nabla_{\boldsymbol{y}} V_{n}^{0}\right\|_{L^{2}\left(\Omega_{0}\right)}\left\|\boldsymbol{z}^{0}-\boldsymbol{w}^{0}\right\|_{H^{1}\left(\Omega_{0}\right)}\|v\|_{H^{1}\left(\Omega_{0}\right)},
\end{aligned}
$$

we get, thanks to 63 and the fact that $\boldsymbol{z}^{0}, \boldsymbol{w}^{0} \in L^{\infty}\left(0, \tau ; H^{1}\left(\Omega_{0}\right)\right)$ and $c^{0} \in$ $L^{\infty}\left(0, \tau ; L^{4}\left(\Omega_{0}\right)\right)$,

$$
\begin{aligned}
\int_{0}^{\tau}\left\|\partial_{t} V_{n}^{0}\right\|_{H^{1}\left(\Omega_{0}\right)^{\prime}}^{2} & \lesssim \int_{0}^{\tau}\left\|\nabla_{\boldsymbol{y}} V_{n}^{0}\right\|_{L^{2}\left(\Omega_{0}\right)}^{2}\left\|\boldsymbol{z}^{0}-\boldsymbol{w}^{0}\right\|_{H^{1}\left(\Omega_{0}\right)}^{2} \\
& +\int_{0}^{\tau}\left\|D \nabla_{\boldsymbol{y}} V_{n}^{0}\right\|_{L^{2}\left(\Omega_{0}\right)}^{2}+\left\|c^{0} V_{n}^{0}\right\|_{L^{2}\left(\Omega_{0}\right)}^{2}+\left\|S^{0}\right\|_{L^{2}\left(\Omega_{0}\right)}^{2} \lesssim 1 .
\end{aligned}
$$

Therefore, up to a subsequence, $\left(\partial_{t} V_{n}^{0}\right)_{n \in \mathbb{N}}$ converges weakly in $L^{2}\left(0, \tau ; H^{1}\left(\Omega_{0}\right)^{\prime}\right)$ to $\partial_{t} V^{0}$. We deduce from this that $V \in \mathscr{C}^{0}\left([0, \tau] ; L^{2}\left(\Omega_{0}\right)\right)$.

We can now pass to the limit in $60-61$ in a similar way as in [14] and prove the existence of a solution to Problem (56)-(58). To obtain its uniqueness, we begin by showing that $V^{0}$ satisfies the maximum principle. After an approximation procedure, we can choose $\zeta^{0}$ as the nonpositive part of $V^{0}$, which we write $\left(V^{0}\right)^{-}$in 59. . For all $t \in(0, \tau)$, we have

$$
\begin{array}{rl}
\left\|\left(V^{0}\right)^{-}(t)\right\|_{L^{2}\left(\Omega_{0}\right)}^{2}+2 \int_{0}^{t} \int_{\Omega_{0}} \mathbf{1}_{V^{0}<0} & \left.J \nabla_{\boldsymbol{y}} V^{0}\right|^{2}+2 \int_{0}^{t} \int_{\Omega_{0}} \mathbf{1}_{V^{0}<0} c^{0}\left|V^{0}\right|^{2} \\
\leq\left\|\left(V^{\mathrm{in}}\right)^{-}\right\|_{L^{2}\left(\Omega_{0}\right)}^{2}-\int_{0}^{t} \int_{\Omega_{0}} S^{0}\left(V^{0}\right)^{-},
\end{array}
$$

where $\mathbf{1}_{A}$ denotes the characteristic function of a subset $A \subset \Omega_{0}$. Since, here, $V^{\text {in }}, c^{0}$ and $S^{0}$ are nonnegative, this yields $V^{0} \geq 0$. The uniqueness of the weak solution to $(56)-(58)$ follows. 
Estimate (54) can be obtained by taking $\zeta=V$ in (53) and applying Grönwall's lemma. For 55 , we can apply 53 to $\zeta \in \mathscr{D}(\widehat{\Omega})$ and reason similarly to what we have done for $\partial_{t} V^{0}$ above, except for the following term, for which we write

$$
\left|\iint_{\widehat{\Omega}} \zeta \boldsymbol{z} \cdot \nabla_{\boldsymbol{x}} V\right| \leq\|\boldsymbol{z}\|_{L^{\infty}\left(0, \tau ; L^{2}\left(\Omega_{t}\right)\right)}\|V\|_{L^{2}\left(0, \tau ; H^{1}\left(\Omega_{t}\right)\right)}\|\zeta\|_{L^{2}\left(0, \tau ; H^{2}\left(\Omega_{t}\right)\right)} .
$$

This concludes the proof of Theorem 3.8 .

\subsection{Application of the Schauder theorem}

Thanks to the results stated in the previous sections, we can check that the map $\Lambda$ defined by (27)-(35) satisfies the hypotheses of the Schauder fixed-point theorem. From Theorems 3.1, 3.4, and 3.8, we deduce that $\Lambda$ is well-defined and that there exists a constant $C>0$ depending only on $\boldsymbol{w}, N, \chi$ and the initial data, such that

$$
\forall(\boldsymbol{u}, Y, \Theta) \in E, \quad\|\Lambda(\boldsymbol{u}, Y, \Theta)\|_{E} \leq C .
$$

\section{Step $1-\Lambda$ is compact}

$$
\text { Let }\left(\boldsymbol{u}_{n}, Y_{n}, \Theta_{n}\right)_{n \in \mathbb{N}} \text { be a bounded sequence of }\left(E,\|\cdot\|_{E}\right) \text {. Let }
$$

$$
\left(\widetilde{\boldsymbol{u}_{n}}, \widetilde{Y_{n}}, \widetilde{\Theta_{n}}\right)_{n \in \mathbb{N}}=\left(\Lambda\left(\boldsymbol{u}_{n}, Y_{n}, \Theta_{n}\right)\right)_{n \in \mathbb{N}}
$$

and let $\left(f_{n}\right)_{n \in \mathbb{N}}$ be the sequence of solutions to the corresponding Vlasov equations. Thanks to Remark 3.3 there exists a compact of $\overline{\widehat{\Omega}} \times \mathbb{R}^{3} \times \mathbb{R}_{+} \times \mathbb{R}_{+}$ that contains all the supports of the functions $f_{n}$. The bounds $40-43$ are therefore uniform with respect to $n \in \mathbb{N}$.

The convergence, up to a subsequence, of $\left(\widetilde{\boldsymbol{u}_{n}}\right)_{n \in \mathbb{N}}$ is a consequence of the Arzelà-Ascoli theorem, which we apply in $\mathscr{C}^{0}\left([0, \tau] ; L^{2}(B)\right)$ for any closed ball $B$ of $\mathbb{R}^{3}$. The sequence is indeed uniformly equicontinuous thanks to the bound 48 and the fact that, for all $\varphi \in \mathscr{C}^{0}\left([0, \tau] ; L^{2}(B)\right)$,

$$
\forall t_{1}, t_{2} \in(0, \tau), \quad\|\varphi\|_{L^{2}(B)}^{2} \leq\left|t_{2}-t_{1}\right|\left\|\partial_{t} \varphi\right\|_{L^{2}\left((0, \tau) \times \mathbb{R}^{3}\right)}^{2} .
$$

Moreover, for all $t \in[0, \tau],\left(\widetilde{\boldsymbol{u}_{n}}(t)\right)_{n \in \mathbb{N}}$ is precompact in $L^{2}(B)$ thanks to the Rellich-Kondrachov theorem. Then there exists $\boldsymbol{u} \in \mathscr{C}^{0}\left([0, \tau] ; L_{\text {loc }}^{2}\left(\mathbb{R}^{3}\right)\right)$ such that the sequence $\left(\widetilde{\boldsymbol{u}_{n}}\right)_{n \in \mathbb{N}}$ converges to $\boldsymbol{u}$ in $\mathscr{C}^{0}\left([0, \tau] ; L^{2}(B)\right)$ for any closed ball $B$ of $\mathbb{R}^{3}$. Thanks to Estimates $(43)$ and $(47)$ and the monotone convergence theorem, $\widetilde{\boldsymbol{u}}$ belongs to $L^{\infty}\left(0, \tau ; L^{2}\left(\mathbb{R}^{3}\right)\right)$. Then the equicontinuity of the sequence $\left(\widetilde{\boldsymbol{u}_{n}}\right)_{n \in \mathbb{N}}$ ensures that $\boldsymbol{u} \in \mathscr{C}^{0}\left([0, \tau] ; L^{2}\left(\mathbb{R}^{3}\right)\right)$. Furthermore, Estimate 477 shows that $\left(\nabla_{\boldsymbol{x}} \widetilde{\boldsymbol{u}_{n}}\right)_{n \in \mathbb{N}}$ is bounded in $L^{2}\left((0, \tau) \times \mathbb{R}^{3}\right)$ and therefore, up to a subsequence, it converges weakly to $\nabla_{\boldsymbol{x}} \widetilde{\boldsymbol{u}}$ in $L^{2}\left((0, \tau) \times \mathbb{R}^{3}\right)$. The weak lower semi-continuity of $\|\cdot\|_{L^{2}\left((0, \tau) \times \mathbb{R}^{3}\right)}$ then implies that $\boldsymbol{u} \in L^{2}\left(0, \tau ; H^{1}\left(\mathbb{R}^{3}\right)\right)$. Finally, we prove that $\left(\widetilde{\boldsymbol{u}}_{n}\right)_{n \in \mathbb{N}}$ converges to $\boldsymbol{u}$ in $E$. For any $R>0$, if we denote 
by $B_{R}$ the ball of $\mathbb{R}^{3}$ centered at 0 and of radius $R$, we have, for all $t \in[0, \tau]$,

$$
\begin{aligned}
\left\|\left(\widetilde{\boldsymbol{u}}(t)-\widetilde{\boldsymbol{u}_{n}}(t)\right) \gamma\right\|_{L^{1}\left(\mathbb{R}^{3}\right)} \leq\left\|\widetilde{\boldsymbol{u}}(t)-\widetilde{\boldsymbol{u}_{n}}(t)\right\|_{L^{2}\left(B_{R}\right)}\|\gamma\|_{L^{2}\left(\mathbb{R}^{3}\right)} \\
+\left\|\widetilde{\boldsymbol{u}}(t)-\widetilde{\boldsymbol{u}_{n}}(t)\right\|_{L^{2}\left(\mathbb{R}^{3}\right)}\left\|\gamma \mathbf{1}_{|x|>R}\right\|_{L^{2}\left(\mathbb{R}^{3}\right)}
\end{aligned}
$$

Thanks to Estimate 477 and the fact that $\gamma \in L^{2}\left(\mathbb{R}^{3}\right)$, we deduce from this that

$$
\lim _{n \rightarrow+\infty} \sup _{t \in[0, \tau]}\left\|\left(\widetilde{\boldsymbol{u}}(t)-\widetilde{\boldsymbol{u}_{n}}(t)\right) \gamma\right\|_{L^{1}\left(\mathbb{R}^{3}\right)}=0 .
$$

We now deal with the convergence of $\left(\widetilde{Y}_{n}\right)_{n \in \mathbb{N}}$, the case of $\left(\widetilde{\Theta_{n}}\right)_{n \in \mathbb{N}}$ being completely similar. We begin by noticing that thanks to Theorem 3.8, for all $n \in \mathbb{N}, \widetilde{Y_{n}}$ is nonnegative and, using the the same argument, since $Y_{v \text {,surf }} \leq 1$, we can prove that $\widetilde{Y_{n}} \leq 1$. In order to get compactness, we apply an AubinLions-type result due to [25, Corollary 1]. Indeed, thanks to the bounds (41) and $54,\left(\widetilde{Y_{n}}\right)_{n \in \mathbb{N}}$ is bounded in $L^{2}\left(0, \tau ; H^{1}\left(\Omega_{t}\right)\right)$. And with $\left.42,, 43,, 47\right)$ and 55 , for all $\zeta \in \mathscr{D}(\widehat{\Omega})$, we obtain

$$
\left|\left\langle\partial_{t} \widetilde{Y_{n}}, \zeta\right\rangle\right| \lesssim_{N}\|\zeta\|_{L^{2}\left(0, \tau ; H^{2}\left(\Omega_{t}\right)\right)} .
$$

This allows to state that, up to a subsequence, $\left(\widetilde{Y_{n}}\right)_{n \in \mathbb{N}}$ converges in $L^{2}(\widehat{\Omega})$.

In conclusion, we have obtained that, up to a subsequence, $\left(\widetilde{\boldsymbol{u}_{n}}, \widetilde{Y_{n}}, \widetilde{\Theta_{n}}\right)_{n \in \mathbb{N}}$ converges in $\left(E,\|\cdot\|_{E}\right)$.

\section{Step $2-\Lambda$ is continuous}

Let $\left(\boldsymbol{u}_{n}, Y_{n}, \Theta_{n}\right)_{n \in \mathbb{N}}$ be a sequence of $E$ converging towards $(\boldsymbol{u}, Y, \Theta)$. It is enough to prove that, from any subsequence of

$$
\left(\widetilde{\boldsymbol{u}_{n}}, \widetilde{Y_{n}}, \widetilde{\Theta_{n}}\right)_{n \in \mathbb{N}}=\left(\Lambda\left(\boldsymbol{u}_{n}, Y_{n}, \Theta_{n}\right)\right)_{n \in \mathbb{N}}
$$

we can extract a subsequence which converges to $(\widetilde{\boldsymbol{u}}, \widetilde{Y}, \widetilde{\Theta})=\Lambda(\boldsymbol{u}, Y, \Theta)$ in $E$. For the sake of clarity, we will not modify the indices when dealing with subsequences. The compactness of $\Lambda$ ensures that $\left(\widetilde{\boldsymbol{u}_{n}}, \widetilde{Y_{n}}, \widetilde{\Theta_{n}}\right)_{n \in \mathbb{N}}$ converges to $(\boldsymbol{U}, H, Z)$ in $E$ up to a subsequence.

The convergences of $\left(\widetilde{\boldsymbol{u}_{n}}\right)_{n \in \mathbb{N}}$ in $\mathscr{C}^{0}\left([0, \tau] \times L_{\text {loc }}^{2}\left(\mathbb{R}^{3}\right)\right)$ and in $w-L^{2}\left((0, \tau) \times \mathbb{R}^{3}\right)$ provide that, with standard arguments, we can take the limit $n \rightarrow+\infty$ in all the terms appearing in 49), except the one on the right-hand side. For this term, Estimate 39 ensures that, up to a subsequence, $\left(\overline{f_{n}}\right)_{n \in \mathbb{N}}$ converges weakly-* in $L^{\infty}\left((0, \tau) \times \mathbb{R}^{3} \times \mathbb{R}^{3} \times \mathbb{R}_{+}^{*} \times \mathbb{R}_{+}^{*}\right)$ and weakly in $L^{p}\left((0, \tau) \times \mathbb{R}^{3} \times \mathbb{R}^{3} \times \mathbb{R}_{+}^{*} \times \mathbb{R}_{+}^{*}\right)$ for all $p \in(1, \infty)$. Furthermore, the convergence of $\left(\boldsymbol{u}_{n}, Y_{n}, \Theta_{n}\right)$ in $E$ provides the convergences of $\left(Y_{n}\right)_{n \in \mathbb{N}}$ and $\left(\Theta_{n}\right)_{n \in \mathbb{N}}$ in $L^{2}(\widehat{\Omega})$. With this, we can take the limit $n \rightarrow+\infty$ in $(18)$. The uniqueness of the weak solution, given by Theorem 3.1 . yields that the limit of $\left(f_{n}\right)_{n \in \mathbb{N}}$ is $f_{\boldsymbol{u}, Y, \Theta}$. In turn, this results to the convergence of the right-hand side term in 49 and, thanks to the uniqueness of the weakin-time solution proved in Theorem 3.6 , we obtain that the whole sequence $\left(\widetilde{\boldsymbol{u}_{n}}\right)_{n \in \mathbb{N}}$ converges to $\boldsymbol{U}=\widetilde{\boldsymbol{u}}$ in $\mathscr{C}^{0}\left([0, \tau] ; L_{\mathrm{loc}}^{2}\left(\mathbb{R}^{3}\right)\right)$. 
We get the convergence in $L^{2}(\widehat{\Omega})$ of $\left(\widetilde{Y_{n}}\right)_{n \in \mathbb{N}}$ and $\left(\widetilde{\Theta_{n}}\right)_{n \in \mathbb{N}}$ towards, respectively, $Z=\widetilde{Y}$ and $H=\widetilde{\Theta}$ in a similar way. As a conclusion, $\Lambda$ is continuous.

\section{Step 3 - Conclusion}

Finally, we can apply Theorem 2.4 to the map $\Lambda$ restricted to the set

$$
\mathscr{C}^{\prime}=\left\{(\boldsymbol{u}, Y, \Theta) \in \mathscr{C},\|(u, \Theta, Y)\|_{E} \leq C\right\},
$$

where $C>0$ has been defined at the beginning of this section. We obtain the existence of $(\boldsymbol{u}, Y, \Theta) \in \mathscr{C}^{\prime}$ such that $\Lambda(\boldsymbol{u}, Y, \Theta)=(\boldsymbol{u}, Y, \Theta)$.

\subsection{Back to the initial system}

Let $\boldsymbol{u}^{\text {in }}, Y^{\text {in }}, \Theta^{\text {in }}, f^{\text {in }}$ satisfy Assumptions $2,4$.

Let $\left(Y_{n}^{\text {in }}\right)_{n \in \mathbb{N}}$ be a sequence of $H^{1}\left(\Omega_{0}\right)$ that converges to $Y^{\text {in }}$ in $L^{2}\left(\Omega_{0}\right)$ and such that $0 \leq Y_{n}^{\text {in }} \leq 1$ and $\left\|Y_{n}^{\text {in }}\right\|_{L^{2}\left(\Omega_{0}\right)} \leq\left\|Y^{\text {in }}\right\|_{L^{2}\left(\Omega_{0}\right)}$ for all $n \in \mathbb{N}$. Similarly, let $\left(\Theta_{n}^{\text {in }}\right)_{n \in \mathbb{N}}$ be a nonnegative sequence of $H^{1}\left(\Omega_{0}\right)$ that converges to $\Theta^{\text {in }}$ and such that $\left\|\Theta_{n}^{\text {in }}\right\|_{L^{2}\left(\Omega_{0}\right)} \leq\left\|\Theta^{\text {in }}\right\|_{L^{2}\left(\Omega_{0}\right)}$ for all $n \in \mathbb{N}$. Finally, let $\left(f_{n}^{\text {in }}\right)_{n \in \mathbb{N}}$ be a nonnegative sequence of $L^{\infty}\left(\Pi_{0}\right)$ such that, for all $n \in \mathbb{N}, f_{n}$ is compactly supported and $f_{n}^{\text {in }} \in L^{\infty}\left(0, \tau ; L^{p}\left(\Pi_{t}\right)\right)$ and such that $\left(f_{n}\right)_{n \in \mathbb{N}}$ converges to $f^{\text {in }}$ in $L^{p}\left(\Pi_{0}\right)$ for all $p \in[1, \infty)$ and $w^{*}-L^{\infty}\left(\Pi_{0}\right)$. We also assume that, for all $n \in \mathbb{N}$, $f_{n}^{\text {in }}(\cdot, \cdot, r, \cdot) \equiv 0$ for almost every $r \in\left[0, r_{\text {drug }}\right]$. Lastly, we choose $f_{n}$ so that

$$
\begin{gathered}
\left\|f_{n}^{\text {in }}\right\|_{L^{\infty}\left(\Pi_{0}\right)} \leq\left\|f^{\text {in }}\right\|_{L^{\infty}\left(\Pi_{0}\right)}, \\
\iint_{\Pi_{0}} r^{3} f_{n}^{\text {in }} \leq \iint_{\Pi_{0}} r^{3} f^{\text {in }}, \\
\iint_{\Pi_{0}} r^{3}|v|^{2} f_{n}^{\text {in }} \leq \iint_{\Pi_{0}} r^{3}|v|^{2} f^{\text {in }}, \\
\iint_{\Pi_{0}}|v|^{2} f_{n}^{\text {in }} \leq \iint_{\Pi_{0}}|v|^{2} f^{\text {in }}, \\
\iint_{\Pi_{0}} r^{3} T^{2} f_{n}^{\text {in }} \leq \iint_{\Pi_{0}} r^{3} T^{2} f^{\text {in }} .
\end{gathered}
$$

Let $\left(\chi_{n}\right)_{n \in \mathbb{N}}$ be a sequence of truncation functions satisfying the same hypotheses as $\chi$ in Section 2. Additionally, we suppose that, for all $n \in \mathbb{N}$, $\left\|\chi_{n}\right\|_{L^{\infty}(\mathbb{R})} \leq n, 0 \leq \chi_{n}^{\prime} \leq 1$, and for all $v \in \mathbb{R},\left|\chi_{n}(v)\right| \leq v$. Finally, we assume that $\chi_{n \mid[-n+1 / n, n-1 / n]}=\operatorname{Id}_{[-n+1 / n, n-1 / n]}$ so that $\left(\chi_{n}\right)_{n \in \mathbb{N}}$ converges uniformly to $\operatorname{Id}_{\mathbb{R}}$.

Using the results of Subsection 3.4 for all $n \in \mathbb{N}$ there exist $\boldsymbol{u}_{n}, Y_{n}, \Theta_{n}, f_{n}$ weakly solving the system

$$
\begin{gathered}
\partial_{t} f_{n}+\boldsymbol{v} \cdot \nabla_{\boldsymbol{x}} f_{n}+\operatorname{div}_{\boldsymbol{v}}\left(\boldsymbol{G}_{1, n} f_{n}\right)+\partial_{r}\left(G_{2, n} f_{n}\right)+\partial_{T}\left(G_{3, n} f_{n}\right)=0 \quad \text { on } \widehat{\Pi}, \\
f_{n}=0 \quad \text { on } \widehat{\Sigma}^{-}
\end{gathered}
$$




$$
\begin{gathered}
\partial_{t} \boldsymbol{u}_{n}+P_{n} \pi_{\mathrm{L}}\left(\operatorname{div}_{\boldsymbol{x}}\left(\boldsymbol{u}_{n} \otimes \boldsymbol{u}_{n}\right)\right)-\Delta_{\boldsymbol{x}} \boldsymbol{u}_{n}+n P_{n} \pi_{\mathrm{L}}\left(\left(\boldsymbol{u}_{n}-\boldsymbol{w}\right) \mathbf{1}_{\widehat{\Omega}^{c}}\right) \\
=P_{n} \pi_{\mathrm{L}} \int_{\mathbb{R}^{3} \times \mathbb{R}_{+}^{*} \times \mathbb{R}_{+}^{*}} r \eta \overline{f_{n}} \chi_{n}\left(\boldsymbol{v}-\boldsymbol{u}_{n}\right) \quad \text { in }(0, \tau) \times \mathbb{R}^{3}, \\
\operatorname{div}_{\boldsymbol{x}} \boldsymbol{u}_{n}=0 \quad \operatorname{in~}(0, \tau) \times \mathbb{R}^{3}, \\
\partial_{t} Y_{n}+\boldsymbol{z}_{n} \cdot \nabla_{\boldsymbol{x}} Y_{n}-\Delta_{\boldsymbol{x}} Y_{n}=\int_{\mathbb{R}^{3} \times \mathbb{R}_{+}^{*} \times \mathbb{R}_{+}^{*}} r \eta\left(Y_{v, \text { surf }}-Y_{n}\right) f_{n} \quad \text { in } \widehat{\Omega}, \\
\nabla_{\boldsymbol{x}} Y_{n} \cdot \boldsymbol{n}_{t}=0 \quad \text { on } \widehat{\Gamma}, \\
\partial_{t} \Theta_{n}+\boldsymbol{z}_{n} \cdot \nabla_{\boldsymbol{x}} \Theta_{n}-\Delta_{\boldsymbol{x}} \Theta_{n}=\int_{\mathbb{R}^{3} \times \mathbb{R}_{+}^{*} \times \mathbb{R}_{+}^{*}} r \eta\left(T-\Theta_{n}\right) f_{n} \quad \text { in } \widehat{\Omega}, \\
\nabla_{\boldsymbol{x}} \Theta_{n} \cdot \boldsymbol{n}_{t}=0 \quad \text { on } \widehat{\Gamma},
\end{gathered}
$$

where

$$
\begin{gathered}
\boldsymbol{z}_{n}=\boldsymbol{w}-\pi_{\Omega_{t}}\left(\left(\boldsymbol{u}_{n}-\boldsymbol{w}\right) \mathbf{1}_{K_{n}}\right) \\
\boldsymbol{G}_{1, n}=\chi_{n}\left(\boldsymbol{u}_{n}-\boldsymbol{v}\right) \frac{\eta}{r^{2}+\frac{r_{\mathrm{drug}}^{3}}{r}}, \quad G_{2, n}=\frac{\overline{Y_{n}}-Y_{v, \text { surf }}}{r} \eta
\end{gathered}
$$

and

$$
G_{3, n}=G_{2, n}+\frac{\overline{\Theta_{n}}-T}{r^{2}} \eta
$$

Moreover, these solutions satisfy the initial conditions

$$
\boldsymbol{u}_{n}(0)=P_{n} \boldsymbol{u}^{\text {in }}, \quad Y_{n}(0)=Y_{n}^{\text {in }}, \quad \Theta_{n}(0)=\Theta_{n}^{\text {in }}, \quad f_{n}(0)=f_{n}^{\text {in }} .
$$

Remark 3.10. Note that, thanks to Theorem 3.6, $\boldsymbol{u}_{n} \in \mathscr{C}^{1}\left([0, \tau] ; \mathscr{F}_{n}\right)$ is a strong solution of the regularized Navier-Stokes equations.

Thanks to Theorem 3.1, Remark 3.2 and Assumption 64, we have

$$
\left\|f_{n}\right\|_{L^{\infty}(\widehat{\Pi})} \lesssim\left\|f_{n}^{\text {in }}\right\|_{L^{\infty}\left(\Pi_{0}\right)} \lesssim 1
$$

Therefore, there exists $f \in L^{\infty}(\widehat{\Pi})$ such that, up to a subsequence, $\left(f_{n}\right)_{n \in \mathbb{N}}$ converges to $f$ weakly-* in $L^{\infty}(\widehat{\Pi})$. Another consequence of $\sqrt{39}$ is the convergence of $\left(f_{n}\right)_{n \in \mathbb{N}}$ weakly in $L^{q}\left(0, \tau ; L^{p}\left(\Pi_{t}\right)\right)$ for all $1<p, q<\infty$. In particular, we have $f \geq 0$.

Estimate (47) on $\boldsymbol{u}_{n}$ is no longer satisfactory since it depends on $n$. We are thus led to write an energy estimate such as (17). Multiplying (71) by $\boldsymbol{u}_{n}$ and integrating over $(0, t) \times \mathbb{R}^{3}$ for $t \in(0, \tau)$ yields

$$
\begin{aligned}
& \frac{1}{2}\left\|\boldsymbol{u}_{n}(t)\right\|_{L^{2}\left(\mathbb{R}^{3}\right)}^{2}+\int_{0}^{t}\left\|\nabla_{\boldsymbol{x}} \boldsymbol{u}_{n}\right\|_{L^{2}\left(\mathbb{R}^{3}\right)}^{2}+n \int_{0}^{t} \int_{\mathbb{R}^{3}} \mathbf{1}_{\widehat{\Omega}^{c}}\left(\boldsymbol{u}_{n}-\boldsymbol{w}\right) \cdot \boldsymbol{u}_{n} \\
& =\int_{0}^{t} \iint_{\mathbb{R}^{3} \times \mathbb{R}^{3} \times \mathbb{R}_{+}^{*} \times \mathbb{R}_{+}^{*}} r \eta \overline{f_{n}} \chi_{n}\left(\boldsymbol{v}-\boldsymbol{u}_{n}\right) \cdot \boldsymbol{u}_{n}+\frac{1}{2}\left\|P_{n} \boldsymbol{u}^{\mathrm{in}}\right\|_{L^{2}\left(\mathbb{R}^{3}\right)}^{2} .
\end{aligned}
$$


Furthermore, thanks to the stability of the renormalized solutions of the Vlasov equation, due to the DiPerna-Lions theory [12], we can deal with the Vlasov equation as if its coefficients and $f_{n}$ were smooth. For more details, the reader can refer to [19, Remark 3.1]. Then, multiplying $[69)$ by $\left(r^{3}+r_{\text {drug }}^{3}\right)|\boldsymbol{v}|^{2} / 2$ and integrating over $\widehat{\Pi}_{t}$ leads to

$$
\begin{aligned}
\frac{1}{2} \iint_{\Pi_{t}}\left(r^{3}+r_{\text {drug }}^{3}\right)|\boldsymbol{v}|^{2} f_{n} \leq \iiint_{\widehat{\Pi}_{t}} r \eta f_{n} \chi_{n}\left(\boldsymbol{u}_{n}-\right. & \boldsymbol{v}) \cdot \boldsymbol{v}+3 \iiint_{\widehat{\Pi}_{t}} r|\boldsymbol{v}|^{2} \eta f_{n} \\
& +\frac{1}{2} \iint_{\Pi_{0}}\left(r^{3}+r_{\text {drug }}^{3}\right)|\boldsymbol{v}|^{2} f_{n}^{\text {in }},
\end{aligned}
$$

because $f_{n}, Y_{n}$ and $Y_{v \text {,surf }}$ are nonnegative, $Y_{n}, Y_{v \text {,surf }} \leq 1$ and $f_{n \mid \widehat{\Sigma}^{-}}=0$. Summing these inequalities, we obtain

$$
\begin{aligned}
& \frac{1}{2}\left\|\boldsymbol{u}_{n}(t)\right\|_{L^{2}\left(\mathbb{R}^{3}\right)}^{2}+\frac{1}{2} \iint_{\Pi_{t}}\left(r^{3}+r_{\mathrm{drug}}^{3}\right)|\boldsymbol{v}|^{2} f_{n}+\int_{0}^{t}\left\|\nabla_{\boldsymbol{x}} \boldsymbol{u}_{n}\right\|_{L^{2}\left(\mathbb{R}^{3}\right)}^{2} \\
& +n \int_{0}^{t} \int_{\mathbb{R}^{3}} \mathbf{1}_{\widehat{\Omega}^{c}}\left(\boldsymbol{u}_{n}-\boldsymbol{w}\right) \cdot \boldsymbol{u}_{n}+\iiint_{\widehat{\Pi}_{t}} r \eta f_{n} \chi\left(\boldsymbol{v}-\boldsymbol{u}_{n}\right) \cdot\left(\boldsymbol{v}-\boldsymbol{u}_{n}\right) \\
& \quad \leq 3 \iiint_{\widehat{\Pi}_{t}} r|\boldsymbol{v}|^{2} \eta f_{n}+\frac{1}{2}\left\|\boldsymbol{u}^{\mathrm{in}}\right\|_{L^{2}\left(\mathbb{R}^{3}\right)}^{2}+\frac{1}{2} \iint_{\Pi_{0}}\left(r^{3}+r_{\mathrm{drug}}^{3}\right)|\boldsymbol{v}|^{2} f^{\text {in }}
\end{aligned}
$$

Since $\chi(\boldsymbol{v}) \cdot \boldsymbol{v} \geq 0$ for all $\boldsymbol{v}$, we only need to replace the unsigned term

$$
n \int_{0}^{t} \int_{\mathbb{R}^{3}} \mathbf{1}_{\widehat{\Omega}^{c}}\left(\boldsymbol{u}_{n}-\boldsymbol{w}\right) \cdot \boldsymbol{u}_{n} \quad \text { by } \quad n \int_{0}^{t} \int_{\mathbb{R}^{3}} \mathbf{1}_{\widehat{\Omega}^{c}}\left|\boldsymbol{u}_{n}-\boldsymbol{w}\right|^{2} .
$$

We proceed in the same way as [8]. By multiplying 771 by $P_{n} \boldsymbol{w}$ and integrating over $(0, t) \times \mathbb{R}^{3}$, we get

$$
\begin{gathered}
-n \int_{0}^{t} \int_{\mathbb{R}^{3}} \mathbf{1}_{\widehat{\Omega}^{c}}\left(\boldsymbol{u}_{n}-\boldsymbol{w}\right) \cdot \boldsymbol{w}=\int_{\mathbb{R}^{3}} \boldsymbol{u}_{n}(t) \cdot \boldsymbol{w}(t)-\int_{\mathbb{R}^{3}} \boldsymbol{u}_{n}^{\text {in }} \cdot \boldsymbol{w}(0) \\
-\int_{0}^{t} \int_{\mathbb{R}^{3}} \boldsymbol{u}_{n} \cdot \partial_{t} \boldsymbol{w}+\int_{0}^{t} \int_{\mathbb{R}^{3}} \nabla_{\boldsymbol{x}} \boldsymbol{u}_{n}: P_{n} \nabla_{\boldsymbol{x}} \boldsymbol{w} \\
\quad+\int_{0}^{t} \int_{\mathbb{R}^{3}}\left(\operatorname{div}_{\boldsymbol{x}}\left(\boldsymbol{u}_{n} \otimes \boldsymbol{u}_{n}\right)\right) \cdot \boldsymbol{w} \\
+\int_{0}^{t} \iiint \int_{\mathbb{R}^{3} \times \mathbb{R}^{3} \times \mathbb{R}_{+}^{*} \times \mathbb{R}_{+}^{*}} r \eta_{r} \theta_{n} \overline{f_{n}} \chi_{n}\left(\boldsymbol{v}-\boldsymbol{u}_{n}\right) \cdot P_{n} \boldsymbol{w} .
\end{gathered}
$$

We then use Cauchy-Swcharz's and Young's inequalities, as well as the fact that 
$\left|\chi_{n}(\boldsymbol{z})\right|^{2} \leq \chi_{n}(\boldsymbol{z}) \cdot \boldsymbol{z}$ for all $\boldsymbol{z} \in \mathbb{R}^{3}$, to find, by summing (??) and 77 ,

$$
\begin{aligned}
& \frac{1}{4}\left\|\boldsymbol{u}_{n}(t)\right\|_{L^{2}\left(\mathbb{R}^{3}\right)}^{2}+\frac{1}{2} \iint_{\Pi_{t}}\left(r^{3}+r_{\mathrm{drug}}^{3}\right)|\boldsymbol{v}|^{2} f_{n} \\
&+\frac{1}{8} \int_{0}^{t}\left\|\nabla_{\boldsymbol{x}} \boldsymbol{u}_{n}\right\|_{L^{2}\left(\mathbb{R}^{3}\right)}^{2}+n \int_{0}^{t} \int_{\mathbb{R}^{3}} \mathbf{1}_{\widehat{\Omega}^{c}}\left|\boldsymbol{u}_{n}-\boldsymbol{w}\right|^{2} \\
& \quad+\frac{1}{2} \iiint_{\widehat{\Pi}_{t}} r \eta f_{n} \chi_{n}\left(\boldsymbol{v}-\boldsymbol{u}_{n}\right) \cdot\left(\boldsymbol{v}-\boldsymbol{u}_{n}\right) \lesssim 1 .
\end{aligned}
$$

Therefore, $\left(\boldsymbol{u}_{n}\right)_{n \in \mathbb{N}}$ is bounded in $L^{\infty}\left(0, \tau ; L^{2}\left(\mathbb{R}^{3}\right)\right) \cap L^{2}\left(0, \tau ; H^{1}\left(\mathbb{R}^{3}\right)\right)$ and there exists $\boldsymbol{u} \in L^{\infty}\left(0, \tau ; L^{2}\left(\mathbb{R}^{3}\right)\right) \cap L^{2}\left(0, \tau ; H^{1}\left(\mathbb{R}^{3}\right)\right)$ such that, up to a subsequence, $\left(\boldsymbol{u}_{n}\right)_{n \in \mathbb{N}}$ converges to $\boldsymbol{u}$ in $w-L^{2}\left(0, \tau ; H^{1}\left(\mathbb{R}^{3}\right)\right)$ and $w^{*}-L^{\infty}\left(0, \tau ; L^{2}\left(\mathbb{R}^{3}\right)\right)$.

We also need a new estimate for $\left(\Theta_{n}\right)_{n \in \mathbb{N}}$. After an approximation procedure, we can take $\zeta=\Theta_{n} \mathbf{1}_{[0, t]}$ in 53 and obtain

$$
\begin{aligned}
\left\|\Theta_{n}(t)\right\|_{L^{2}\left(\Omega_{t}\right)}^{2}+2 \int_{0}^{t}\left\|\nabla_{\boldsymbol{x}} \Theta_{n}\right\|_{L^{2}\left(\Omega_{s}\right)}^{2}+2 \iiint_{\widehat{\Pi}_{t}} r\left(\Theta_{n}-T\right) \Theta_{n} \eta f_{n} & \\
= & =\left\|T_{n}^{\text {in }}\right\|_{L^{2}\left(\Omega_{0}\right)}^{2} .
\end{aligned}
$$

Furthermore, multiplying 69 by $r^{3} T^{2}$ and integrating over $\widehat{\Pi}_{t}$ yields, thanks to the boundary condition 70 and the fact that $0 \leq Y_{n}, Y_{v \text {,surf }} \leq 1$ and $f_{n} \geq 0$,

$$
\begin{aligned}
\iint_{\Pi_{t}} r^{3} T^{2} f_{n}(t) \leq 6 \iiint_{\widehat{\Pi}_{t}} r T^{2} \eta f_{n} & +4 \iiint_{\widehat{\Pi}_{t}} r T \eta f_{n} \\
& +2 \iiint_{\widehat{\Pi}_{t}} r \chi_{n}\left(\Theta_{n}-T\right) T \eta f_{n}+\iint_{\Pi_{0}} r^{3} T^{2} f_{n}^{\text {in }} .
\end{aligned}
$$

Summing these inequalities leads to

$$
\begin{aligned}
\left\|\Theta_{n}(t)\right\|_{L^{2}\left(\Omega_{t}\right)}^{2} & +\iint_{\Pi_{t}} r^{3} T^{2} f_{n}(t) \\
& +2 \int_{0}^{t}\left\|\nabla_{\boldsymbol{x}} \Theta_{n}\right\|_{L^{2}\left(\Omega_{s}\right)}^{2}+2 \iiint_{\widehat{\Pi}_{t}} r\left|\Theta_{n}-T\right|^{2} \eta f_{n} \\
\leq & 6 \iiint_{\widehat{\Pi}_{t}} r T^{2} \eta f_{n}+4 \iiint_{\widehat{\Pi}_{t}} r T \eta f_{n}+\iint_{\Pi_{0}} r^{3} T^{2} f_{n}^{\text {in }} \\
& \quad 2 \iiint_{\widehat{\Pi}_{t}} r T\left[\chi_{n}\left(\Theta_{n}-T\right)-\left(\Theta_{n}-T\right)\right] \eta f_{n} .
\end{aligned}
$$

As in the introduction, we deal with the first two terms of the right-hand side by ensuring, thanks to the Grönwall lemma again, that

$$
\iint_{\Pi_{t}} r^{3} f_{n}(t) \lesssim 1
$$


Since $\left|\chi_{n}\left(\Theta_{n}-T\right)-\left(\Theta_{n}-T\right)\right| \leq 2\left|\Theta_{n}-T\right| \mathbf{1}_{\left|\Theta_{n}-T\right|>n-1 / n}$ and thanks to the Young inequality and the definition of $\eta$, we also have

$$
\begin{aligned}
\iiint_{\widehat{\Pi}_{t}} r T\left[\chi_{n}\left(\Theta_{n}-T\right)-\right. & \left.\left(\Theta_{n}-T\right)\right] \eta f_{n} \\
& \leq 2 \iiint_{\widehat{\Pi}_{t}} r T|\Theta-T| \eta f_{n} \\
& \leq \frac{4}{r_{\text {drug }}} \iiint_{\widehat{\Pi}_{t}} r^{3} T^{2} f_{n}+\frac{1}{2} \iiint_{\widehat{\Pi}_{t}} r\left|\Theta_{n}-T\right|^{2} \eta f_{n} .
\end{aligned}
$$

Then, 79 leads to

$$
\iint_{\Pi_{t}} r^{3} T^{2} f_{n} \lesssim 1
$$

thanks to the Grönwall lemma again. Note that by a straightforward application of Fatou's lemma, 80, and 82 yield

$$
\iint_{\Pi_{t}} r^{3} T^{2} f<\infty, \quad \iint_{\Pi_{t}} r^{3} f<\infty
$$

As a conclusion, we can write

$$
\begin{aligned}
\left\|\Theta_{n}(t)\right\|_{L^{2}\left(\Omega_{t}\right)}^{2}+\iint_{\Pi_{t}} r^{3} T^{2} f_{n}(t)+2 \int_{0}^{t}\left\|\nabla_{\boldsymbol{x}} \Theta_{n}\right\|_{L^{2}\left(\Omega_{s}\right)}^{2} & \\
& \quad+\iiint_{\widehat{\Pi}_{t}} r\left|\Theta_{n}-T\right|^{2} \eta f_{n} \lesssim 1 .
\end{aligned}
$$

Let $\mathcal{B}$ be an open ball such that, for every $t \in[0, \tau], \overline{\Omega_{t}} \subset \mathcal{B}$. We define respectively $\left(\overline{\Theta_{n}}\right)_{n \in \mathbb{N}},\left(\overline{\nabla_{\boldsymbol{x}} \Theta_{n}}\right)_{n \in \mathbb{N}}$ as the sequence of continuations of $\left(\Theta_{n}\right)_{n \in \mathbb{N}}$ and $\left(\nabla_{x} \Theta_{n}\right)_{n \in \mathbb{N}}$ by 0 on $\left(\mathbb{R}_{+}^{*} \times \mathcal{B}\right) \backslash \widehat{\Omega}$. From Estimate $\sqrt{83}$, we infer that $\left(\overline{\Theta_{n}}\right)_{n \in \mathbb{N}}$ is bounded in $L^{\infty}\left(\mathbb{R}_{+}^{*} ; L^{2}(\mathcal{B})\right) \cap L^{2}\left(\mathbb{R}_{+}^{*} ; H^{1}(\mathcal{B})\right)$. Therefore, there exists $\Theta \in L^{\infty}\left(\mathbb{R}_{+} ; L^{2}(\mathcal{B})\right) \cap L^{2}\left(\mathbb{R}_{+} ; H^{1}(\mathcal{B})\right)$ such that, up to a subsequence, $\left(\overline{\Theta_{n}}\right)_{n \in \mathbb{N}}$ converges to $\Theta$ in $w-L^{2}\left(\mathbb{R}_{+} ; H^{1}(\mathcal{B})\right)$ and $w^{*}-L^{\infty}\left(\mathbb{R}_{+} ; L^{2}(\mathcal{B})\right)$.

In the same way, we obtain an estimate on $\left(Y_{n}\right)_{n \in \mathbb{N}}$. Choosing $\zeta=Y_{n} \mathbf{1}_{[0, t]}$ in (3.7), we get, since $0 \leq Y_{n}, Y_{v \text {,surf }} \leq 1$,

$$
\frac{1}{2}\left\|Y_{n}(t)\right\|_{L^{2}\left(\Omega_{t}\right)}^{2}+\int_{0}^{t}\left\|\nabla_{\boldsymbol{x}} Y_{n}\right\|_{L^{2}\left(\Omega_{s}\right)}^{2} \leq \iiint_{\widehat{\Pi}_{t}} r \eta f_{n}+\frac{1}{2}\left\|Y_{n}^{\mathrm{in}}\right\|_{L^{2}\left(\Omega_{0}\right)}^{2},
$$

hence, using 80,

$$
\frac{1}{2}\left\|Y_{n}(t)\right\|_{L^{2}\left(\Omega_{t}\right)}^{2}+\int_{0}^{t}\left\|\nabla_{\boldsymbol{x}} Y_{n}\right\|_{L^{2}\left(\Omega_{s}\right)}^{2} \lesssim 1 .
$$

Therefore, $\left(\overline{Y_{n}}\right)_{n \in \mathbb{N}}$ is bounded in $L^{\infty}\left(\mathbb{R}_{+}^{*} ; L^{2}(\mathcal{B})\right) \cap L^{2}\left(\mathbb{R}_{+}^{*} ; H^{1}(\mathcal{B})\right)$ and there exists $Y \in L^{\infty}\left(\mathbb{R}_{+} ; L^{2}(\mathcal{B})\right) \cap L^{2}\left(\mathbb{R}_{+} ; H^{1}(\mathcal{B})\right)$ such that, up to a subsequence, $\left(\overline{Y_{n}}\right)_{n \in \mathbb{N}}$ converges to $Y$ in $w-L^{2}\left(\mathbb{R}_{+} ; H^{1}(\mathcal{B})\right)$ and $w^{*}-L^{\infty}\left(\mathbb{R}_{+} ; L^{2}(\mathcal{B})\right)$. 
The weak convergences we have obtained are enough to justify the asymptotics $n \rightarrow+\infty$ in the linear terms appearing in (18)- 21). For the other terms, we will need to prove strong compactness for $\left(\boldsymbol{u}_{n}\right)_{n \in \mathbb{N}},\left(Y_{n}\right)_{n \in \mathbb{N}}$ and $\left(\Theta_{n}\right)_{n \in \mathbb{N}}$.

Let us first consider the sequence $\left(\boldsymbol{u}_{n}\right)_{n \in \mathbb{N}}$. We apply Theorem 4 of [25] which is an Aubin-Lions type of result specifically engineered to deal with the NavierStokes equation in a time-dependent domain. Namely, under the additional hypotheses that the sequence of normal traces of $\boldsymbol{u}_{n}$ in $L^{2}\left(0, \tau ; H^{-1 / 2}\left(\Omega_{t}\right)\right)$ is compact and that we have a control of the action of $\partial_{t} \boldsymbol{u}_{n}$ on test functions with divergence equal to zero, the sequence $\left(u_{n}\right)_{n \in \mathbb{N}}$ converges in $L^{2}(\widehat{\Omega})$ up to a subsequence.

The first hypothesis is verified since, thanks to $(78),\left(\boldsymbol{u}_{n}\right)_{n \in \mathbb{N}}$ converges to $\boldsymbol{w}$ in $L^{2}\left(\widehat{\Omega}^{c}\right)$ and the normal trace operator on $\widehat{\Gamma}$ is continuous from $\{\boldsymbol{\varphi} \in$ $\left.L^{2}\left(\widehat{\Omega}^{c}\right), \operatorname{div}_{\boldsymbol{x}} \varphi \in L^{2}\left(\widehat{\Omega}^{c}\right)\right\}$ to $L^{2}\left(0, \tau ; H^{-1 / 2}\left(\Omega_{t}\right)\right)$. Note that this implies $\boldsymbol{u}-$ $\boldsymbol{w} \in \mathcal{V}_{0}$.

For the second one, we can prove that, for any $\varphi \in \mathscr{D}(\widehat{\Omega})$ such that $\operatorname{div}_{x} \varphi=$ 0 , we have

$$
\left|\int_{0}^{t} \int_{\Omega_{t}} \partial_{t} \boldsymbol{u}_{n} \cdot \boldsymbol{\varphi}\right| \lesssim\|\varphi\|_{L^{2}\left(0, \tau ; H^{2}\left(\Omega_{t}\right)\right)} .
$$

This is obtained thanks to the Hölder and Gagliardo-Nirenberg inequalities, the continuous injection $H^{2}\left(\Omega_{t}\right) \hookrightarrow L^{\infty}\left(\Omega_{t}\right)$, and Estimate $\sqrt{78)}$. We only detail one computation: since $|\chi(\boldsymbol{v})|^{2} \leq \chi(\boldsymbol{v}) \cdot \boldsymbol{v}$ for all $\boldsymbol{v} \in \mathbb{R}^{3}$, the Cauchy-Schwarz inequality yields

$$
\begin{aligned}
& \mid \iiint_{\widehat{\Pi}} r \eta f_{n} \chi_{n}\left(\boldsymbol{v}-\boldsymbol{u}_{n}\right) \cdot \boldsymbol{\varphi}\left|\leq \int_{0}^{\tau}\|\boldsymbol{\varphi}\|_{H^{2}\left(\Omega_{t}\right)} \iint_{\Pi_{t}} r \eta f_{n}\right| \chi_{n}\left(\boldsymbol{v}-\boldsymbol{u}_{n}\right) \mid \\
& \leq \int_{0}^{\tau}\|\boldsymbol{\varphi}\|_{H^{2}\left(\Omega_{t}\right)}\left(\iint_{\Pi_{t}} r \eta f_{n}\right)^{1 / 2}\left(\iint_{\Pi_{t}} r \eta f_{n}\left|\chi_{n}\left(\boldsymbol{v}-\boldsymbol{u}_{n}\right)\right|^{2}\right)^{1 / 2} \\
& \leq\left(\sup _{t \in(0, \tau)} \iint_{\Pi_{t}} r^{3} f_{n}\right)^{1 / 2}\left(\iiint_{\widehat{\Pi}} r \eta f_{n} \chi_{n}\left(\boldsymbol{v}-\boldsymbol{u}_{n}\right) \cdot\left(\boldsymbol{v}-\boldsymbol{u}_{n}\right)\right)^{1 / 2} \\
& \times\|\boldsymbol{\varphi}\|_{L^{2}\left(0, \tau ; H^{2}\left(\Omega_{t}\right)\right)} \quad \lesssim\|\boldsymbol{\varphi}\|_{L^{2}\left(0, \tau ; H^{2}\left(\Omega_{t}\right)\right)}
\end{aligned}
$$

thanks to $(78)$ and $(80)$. Therefore, up to a subsequence, $\left(\boldsymbol{u}_{n}\right)_{n \in \mathbb{N}}$ converges to $\boldsymbol{u}$ in $L^{2}(\widehat{\Omega})$.

To obtain compactness for the sequence $\left(\Theta_{n}\right)_{n \in \mathbb{N}}$, we apply Corollary 1 of 25]. Since the sequence is bounded in $L^{2}\left(0, \tau ; H^{1}\left(\Omega_{t}\right)\right)$, we just have to prove that, for all $\zeta \in \mathscr{D}(\widehat{\Omega})$,

$$
\iint_{\widehat{\Omega}} \Theta_{n} \partial_{t} \zeta \lesssim\|\zeta\|_{L^{2}\left(0, \tau ; H^{2}\left(\Omega_{t}\right)\right)} .
$$

We do not detail the proof since the right-hand side term of the equation is dealt with in the same way as for $\left(\boldsymbol{u}_{n}\right)_{n \in \mathbb{N}}$ by using of $(80)$ and (83). Therefore, up to 
a subsequence, $\left(\Theta_{n}\right)_{n \in \mathbb{N}}$ converges to $\Theta$ in $L^{2}(\widehat{\Omega})$. The same goes for $\left(Y_{n}\right)_{n \in \mathbb{N}}$, converging to $Y$ in $L^{2}(\widehat{\Omega})$ up to a subsequence.

This allows to take the limit $n \rightarrow+\infty$ in all the nonlinear terms that appear in Definition 2.2. We focus on the only difficulty arising in the asymptotics in the coupling terms from the right-hand sides of the equations. We tackle this case for the Navier-Stokes equation but a similar treatment provides the expected results for the convection-diffusion equations.

Thanks to the strong convergence of $\left(\boldsymbol{u}_{n}\right)_{n \in \mathbb{N}}$, we obtain the $L^{2}$ convergence of $\left(r \eta \chi_{n}\left(\boldsymbol{v}-\boldsymbol{u}_{n}(t)\right)\right)_{n \in \mathbb{N}}$ in a bounded subset of $\Omega_{t} \times \mathbb{R}^{3} \times \mathbb{R}_{+}^{*} \times \mathbb{R}_{+}^{*}$ for almost every $t$. Indeed, let $R, \theta>0$ and $K_{\boldsymbol{v}}$ a compact of $\mathbb{R}^{3}$. Up to a subsequence, we can assume that $\left(\boldsymbol{u}_{n}\right)_{n \in \mathbb{N}}$ converges almost everywhere to $\boldsymbol{u} \in L^{2}(\widehat{\Omega})$. Since $\left(\boldsymbol{u}_{n}(t)\right)_{n \in \mathbb{N}}$ converges to $\boldsymbol{u}(t)$ in $L^{2}\left(\Omega_{t}\right)$ for almost every $t$, the Vitali convergence theorem ensures that $\left(\left|\boldsymbol{u}_{n}(t)\right|^{2}\right)_{n \in \mathbb{N}}$ is uniformly integrable. Since $\left|\chi_{n}\left(\boldsymbol{v}-\boldsymbol{u}_{n}(t)\right)\right| \leq|\boldsymbol{v}|+\left|\boldsymbol{u}_{n}(t)\right|$ and $K_{\boldsymbol{v}}$ is bounded, we deduce from this that $\left(\left|\chi_{n}\left(\boldsymbol{v}-\boldsymbol{u}_{n}(t)\right)\right|^{2}\right)_{n \in \mathbb{N}}$ is uniformly integrable for almost every $t$. Therefore, since this sequence converges almost everywhere, the Vitali convergence theorem ensures the convergence of $\left(\chi_{n}\left(\boldsymbol{v}-\boldsymbol{u}_{n}(t)\right)\right)_{n \in \mathbb{N}}$ to $\boldsymbol{v}-\boldsymbol{u}(t)$ in $L^{2}\left(\Omega_{t} \times K_{\boldsymbol{v}}\right)$ for almost every $t$. This leads to $\left(r \eta \chi_{n}\left(\boldsymbol{v}-\boldsymbol{u}_{n}(t)\right)\right)_{n \in \mathbb{N}}$ converging to $(r \eta(\boldsymbol{v}-\boldsymbol{u}(t)))_{n \in \mathbb{N}}$ in $L^{2}\left(\Omega_{t} \times K_{\boldsymbol{v}} \times[0, R] \times[0, \theta]\right)$ for almost every $t \in(0, \tau)$.

Let $\varphi \in \mathscr{D}(\widehat{\Omega})$. Thanks to the bounds $(78)$ and $(82)$, we can prove that the following convergence occurs uniformly with respect to $n \in \mathbb{N}$ :

$$
\begin{aligned}
\iiint_{\widehat{\Pi}} \mathbf{1}_{|\boldsymbol{v}| \leq M} \mathbf{1}_{r \leq M} \mathbf{1}_{T \leq M} r \eta f_{n} \chi_{n}\left(\boldsymbol{v}-\boldsymbol{u}_{n}\right) & \cdot \boldsymbol{\varphi} \\
& \underset{M \rightarrow+\infty}{\longrightarrow} \iiint_{\widehat{\Pi}} r \eta f_{n} \chi_{n}\left(\boldsymbol{v}-\boldsymbol{u}_{n}\right) \cdot \boldsymbol{\varphi} .
\end{aligned}
$$

Therefore,

$$
\begin{aligned}
\lim _{M \rightarrow+\infty} \lim _{n \rightarrow+\infty} & \iiint_{\widehat{\Pi}} \mathbf{1}_{|\boldsymbol{v}| \leq M} \mathbf{1}_{r \leq M} r \eta f_{n} \chi_{n}\left(\boldsymbol{v}-\boldsymbol{u}_{n}\right) \cdot \boldsymbol{\varphi} \\
& =\lim _{n \rightarrow+\infty} \lim _{M \rightarrow+\infty} \iiint_{\widehat{\Pi}} \mathbf{1}_{|\boldsymbol{v}| \leq M} \mathbf{1}_{r \leq M} \mathbf{1}_{T \leq M} r \eta f_{n} \chi_{n}\left(\boldsymbol{v}-\boldsymbol{u}_{n}\right) \cdot \boldsymbol{\varphi} .
\end{aligned}
$$

Then we deduce from the local convergence we have just obtained, the dominated convergence theorem and the weak convergence of $\left(f_{n}\right)_{n \in \mathbb{N}}$ that

$$
\begin{aligned}
\iiint_{\widehat{\Pi}} \mathbf{1}_{|\boldsymbol{v}| \leq M} \mathbf{1}_{r \leq M} \mathbf{1}_{T \leq M} r \eta f_{n} \chi_{n}\left(\boldsymbol{v}-\boldsymbol{u}_{n}\right) \cdot \boldsymbol{\varphi} \\
\stackrel{{ }_{n \rightarrow+\infty}}{\longrightarrow} \iiint_{\widehat{\Pi}} \mathbf{1}_{|\boldsymbol{v}| \leq M} \mathbf{1}_{r \leq M} \mathbf{1}_{T \leq M} r \eta f(\boldsymbol{v}-\boldsymbol{u}) \cdot \boldsymbol{\varphi} .
\end{aligned}
$$

Besides, thanks to $(78), 80)$ and Fatou's lemma, we can prove that $r \eta f(\boldsymbol{v}-\boldsymbol{u}) \in$ $L^{1}(\widehat{\Pi})$, which allows to take the limit $M \rightarrow+\infty$ and obtain the convergence

$$
\iiint_{\widehat{\Pi}} r \eta f_{n} \chi_{n}\left(\boldsymbol{v}-\boldsymbol{u}_{n}\right) \cdot \varphi \underset{n \rightarrow+\infty}{\longrightarrow} \iiint_{\widehat{\Pi}} r \eta f(\boldsymbol{v}-\boldsymbol{u}) \cdot \varphi .
$$


As a conclusion, we have obtained that $\boldsymbol{u}, Y, \Theta$ and $f$ weakly solves a system of equations similar to Problem (2)-(14). We have yet to deal with factor $\eta$ still appearing in these equations. Namely, we finally prove that under the assumption that $f^{\text {in }}(\cdot, \cdot, r, \cdot) \equiv 0$ for almost every $r \in\left[0, r_{\text {drug }}\right]$, then $f(\cdot, \cdot, \cdot, r, \cdot) \equiv 0$ for $r \in\left[0, r_{\text {drug }}\right]$, so that $\eta f=f$. We use again the DiPerna-Lions theory [12] which enables us, thanks to the uniqueness of the solution of the Vlasov equation, to consider a sequence of solutions $\left(f_{n}\right)_{n \in \mathbb{N}}$ strongly converging to $f$ and for which the coefficients of the equation are smooth. We can thus assume that the functions $\left(f_{n}^{\text {in }}\right)_{n \in \mathbb{N}},\left(\boldsymbol{u}_{n}\right)_{n \in \mathbb{N}},\left(Y_{n}\right)_{n \in \mathbb{N}}$ and $\left(\Theta_{n}\right)_{n \in \mathbb{N}}$ are smooth and that $Y_{n} \geq 0$ for all $n \in \mathbb{N}$ and $f_{n}^{\text {in }}(\cdot, \cdot, r, \cdot) \equiv 0$ for every $r \in\left[0, r_{\text {drug }}\right]$. We also take a sequence $\left(\varepsilon_{n}\right)_{n \in \mathbb{N}}$ of $(0,1)$ with limit 0 and replace $Y_{n}$ by $Y_{n}+\varepsilon_{n}$ in the part of the Vlasov equation that describes radius evolution. With these assumptions, the characteristic curves are defined by

$$
\left\{\begin{array}{l}
\dot{\boldsymbol{X}}_{n}\left(t ; t_{0}, \boldsymbol{x}, \boldsymbol{v}, r, T\right)=\boldsymbol{V}_{n}, \\
\dot{\boldsymbol{V}}_{n}\left(t ; t_{0}, \boldsymbol{x}, \boldsymbol{v}, r, T\right)=\frac{R_{n} \eta\left(R_{n}\right)}{R_{n}^{3}+r_{\mathrm{drug}}^{3}} \chi_{n}\left(\boldsymbol{u}_{n}\left(t, \boldsymbol{X}_{n}\right)-\boldsymbol{V}_{n}\right), \\
\dot{R}_{n}\left(t ; t_{0}, \boldsymbol{x}, \boldsymbol{v}, r, T\right)_{n}=\frac{Y_{n}\left(t, \boldsymbol{X}_{n}\right)+\varepsilon_{n}-Y_{v, \text { surf }}\left(R_{n}, H_{n}\right)}{R_{n}} \eta\left(R_{n}\right), \\
\dot{H}_{n}\left(t ; t_{0}, \boldsymbol{x}, \boldsymbol{v}, r, T\right)_{n}=\frac{Y_{n}\left(t, \boldsymbol{X}_{n}\right)-Y_{v, \text { surf }}\left(R_{n}, H_{n}\right)}{R_{n}^{2}} \eta\left(R_{n}\right)+\frac{\Theta_{n}-H_{n}}{R_{n}^{2}} \eta\left(R_{n}\right), \\
\left(\boldsymbol{X}_{n}, \boldsymbol{V}_{n}, R_{n}, H_{n}\right)\left(t_{0} ; t_{0}, \boldsymbol{x}, \boldsymbol{v}, r, T\right)=(\boldsymbol{x}, \boldsymbol{v}, r, T),
\end{array}\right.
$$

where $(\boldsymbol{x}, \boldsymbol{v}, r, T) \in \mathbb{R}^{3} \times \mathbb{R}^{3} \times \mathbb{R}_{+}^{*} \times \mathbb{R}_{+}^{*}$ and $t_{0} \in[0, \tau)$. Thanks to the CauchyLipschitz theorem, the previous differential system has a unique global solution $\boldsymbol{Z}_{n}=\left(\boldsymbol{X}_{n}, \boldsymbol{V}_{n}, R_{n}, H_{n}\right)$.

Let $n \in \mathbb{N}$. Assume that $r>r_{\text {drug }}$, let $t_{*}=\inf \left\{t \geq t_{0}, R_{n}\left(t ; t_{0}, \boldsymbol{x}, \boldsymbol{v}, r, T\right)=\right.$ $\left.r_{\text {drug }}\right\}$ and suppose that $t_{*}<\tau$. Since $Y_{v \text {,surf }}\left(r_{\text {drug }}, \theta\right)=0$ for all $\theta>0$, $Y_{n} \geq 0$ and $\varepsilon_{n}>0$, we have $\dot{R}_{n}\left(t_{*} ; t_{0}, \boldsymbol{x}, \boldsymbol{v}, r, T\right)>0$, which contradicts the definition of $t_{*}$ since $R_{n}\left(\cdot ; t_{0}, \boldsymbol{x}, \boldsymbol{v}, r, T\right)$ is continuous. Therefore, if $r>r_{\mathrm{drug}}$, then $R_{n}\left(t ; t_{0}, \boldsymbol{x}, \boldsymbol{v}, r, T\right)>r_{\text {drug }}$ for all $t \geq t_{0}$.

Let $(t, \boldsymbol{x}, \boldsymbol{v}, r, T) \in \widehat{\Pi}$, with $r \leq r_{\text {drug. }}$. By the methods of characteristics, there exists a function $J_{n}$ such that, for every $\left(t, \boldsymbol{x}^{\prime}, \boldsymbol{v}^{\prime}, r^{\prime}, T^{\prime}\right) \in \widehat{\Pi}$,

$$
f_{n}\left(t, Z\left(t ; 0, \boldsymbol{x}^{\prime}, \boldsymbol{v}^{\prime}, r^{\prime}, T^{\prime}\right)\right)=f_{n}^{\text {in }}\left(\boldsymbol{x}^{\prime}, \boldsymbol{v}^{\prime}, r^{\prime}, T^{\prime}\right) J_{n}\left(t ; 0, \boldsymbol{x}^{\prime}, \boldsymbol{v}^{\prime}, r^{\prime}, T^{\prime}\right) .
$$

Therefore, with $\left(\boldsymbol{x}^{\prime}, \boldsymbol{v}^{\prime}, r^{\prime}, T^{\prime}\right)=\boldsymbol{Z}_{n}(0 ; t, \boldsymbol{x}, \boldsymbol{v}, r, T)$, we get

$$
f_{n}(t, \boldsymbol{x}, \boldsymbol{v}, r, T)=f_{n}^{\text {in }}\left(\boldsymbol{Z}_{n}(0 ; t, \boldsymbol{x}, \boldsymbol{v}, r, T)\right) J_{n}\left(t, \boldsymbol{Z}_{n}(0 ; t, \boldsymbol{x}, \boldsymbol{v}, r, T)\right) .
$$

If we had $R_{n}(0 ; t, \boldsymbol{x}, \boldsymbol{v}, r, T)>r_{\mathrm{drug}}$, then, as proved in the last paragraph, we would have $r=R_{n}(t ; t, \boldsymbol{x}, \boldsymbol{v}, r, T)>r_{\mathrm{drug}}$. By contradiction, we get $R_{n}(0 ; t, \boldsymbol{x}, \boldsymbol{v}, r, T) \leq$ $r_{\text {drug }}$ and the hypothesis on $f_{n}^{\text {in }}$ yields

$$
f_{n}(t, \boldsymbol{x}, \boldsymbol{v}, r, T)=0 .
$$


Consequently, for all $n \in \mathbb{N}$ and $r \in\left[0, r_{\text {drug }}\right], f_{n}(\cdot, \cdot, \cdot, r, \cdot) \equiv 0$. The weak convergence of $\left(f_{n}\right)_{n \in \mathbb{N}}$ yields the same property for $f$. Therefore, $\eta f=f$, which concludes the proof of Theorem 2.3 .

\section{References}

[1] O. Anoshchenko and A. Boutet de Monvel-Berthier. The existence of the global generalized solution of the system of equations describing suspension motion. Math. Methods Appl. Sci., 20(6):495-519, 1997.

[2] C. Baranger, L. Boudin, P.-E. Jabin, and S. Mancini. A modeling of biospray for the upper airways. In CEMRACS 2004-mathematics and applications to biology and medicine, volume 14 of ESAIM Proc., pages 41-47. EDP Sci., Les Ulis, 2005.

[3] C. Baranger and L. Desvillettes. Coupling Euler and Vlasov equations in the context of sprays: the local-in-time, classical solutions. J. Hyperbolic Differ. Equ., 3(1):1-26, 2006.

[4] F. F. Bonsall. Lectures on some fixed point theorems of functional analysis. Notes by K. B. Vedak. Tata Institute of Fundamental Research, Bombay, 1962.

[5] L. Boudin, L. Desvillettes, C. Grandmont, and A. Moussa. Global existence of solutions for the coupled Vlasov and Navier-Stokes equations. Differential Integral Equations, 22(11-12):1247-1271, 2009.

[6] L. Boudin, C. Grandmont, B. Grec, S. Martin, A. Mecherbet, and F. Noël. Fluid-kinetic modelling for respiratory aerosols with variable size and temperature. To appear in ESAIM Proc. HAL-Id hal-02092574.

[7] L. Boudin, C. Grandmont, A. Lorz, and A. Moussa. Modelling and numerics for respiratory aerosols. Commun. Comput. Phys., 18(3):723-756, 2015 .

[8] L. Boudin, C. Grandmont, and A. Moussa. Global existence of solutions to the incompressible Navier-Stokes-Vlasov equations in a time-dependent domain. J. Differential Equations, 262(3):1317-1340, 2017.

[9] M. Chae, K. Kang, and J. Lee. Global classical solutions for a compressible fluid-particle interaction model. J. Hyperbolic Differ. Equ., 10(3):537-562, 2013.

[10] Y.-P. Choi and J. Jung. Asymptotic analysis for Vlasov-FokkerPlanck/compressible Navier-Stokes equations with a density-dependent viscosity. ArXiv e-prints, page arXiv:1901.01221, Jan 2019. 
[11] L. Desvillettes. Some aspects of the modeling at different scales of multiphase flows. Comput. Methods Appl. Mech. Engrg., 199(21-22):1265-1267, 2010 .

[12] R. J. DiPerna and P.-L. Lions. Ordinary differential equations, transport theory and Sobolev spaces. Invent. Math., 98(3):511-547, 1989.

[13] G. Dufour and P. Villedieu. A second-order multi-fluid model for evaporating sprays. M2AN Math. Model. Numer. Anal., 39(5):931-963, 2005.

[14] L. C. Evans. Partial differential equations, volume 19 of Graduate Studies in Mathematics. American Mathematical Society, Providence, RI, second edition, 2010.

[15] T. Gemci, T. Corcoran, and N. Chigier. A numerical and experimental study of spray dynamics in a simple throat model. Aerosol Sci. Technol., $36: 18-38,2002$.

[16] O. Glass, D. Han-Kwan, and A. Moussa. The Vlasov-Navier-Stokes system in a 2D pipe: existence and stability of regular equilibria. Arch. Ration. Mech. Anal., 230(2):593-639, 2018.

[17] K. Hamdache. Global existence and large time behaviour of solutions for the Vlasov-Stokes equations. Japan J. Indust. Appl. Math., 15(1):51-74, 1998.

[18] D. Han-Kwan, É. Miot, A. Moussa, and I. Moyano. Uniqueness of the solution to the 2D Vlasov-Navier-Stokes system. Rev. Mat. Iberoam., 36(1):3760,2020 .

[19] D. Han-Kwan, A. Moussa, and I. Moyano. Large time behavior of the Vlasov-Navier-Stokes system on the torus. To appear in Arch. Ration. Mech. Anal.

[20] F. Li, Y. Mu, and D. Wang. Global well-posedness and large time behavior of strong solution to a kinetic-fluid model. ArXiv e-prints, aug 2015.

[21] P. W. Longest and M. Hindle. Numerical model to characterize the size increase of combination drug and hygroscopic excipient nanoparticle aerosols. Aerosol Sci. Tech., 45(7):884-899, 2011.

[22] J. Mathiaud. Local smooth solutions of a thin spray model with collisions. Math. Models Methods Appl. Sci., 20(2):191-221, 2010.

[23] A. Mellet and A. Vasseur. Global weak solutions for a Vlasov-FokkerPlanck/Navier-Stokes system of equations. Math. Models Methods Appl. Sci., 17(7):1039-1063, 2007.

[24] A. Mellet and A. Vasseur. Asymptotic analysis for a Vlasov - Fokker Planck / compressible Navier-Stokes system of equations. Comm. Math. Phys., 281(3):573-596, 2008. 
[25] A. Moussa. Some variants of the classical Aubin-Lions Lemma. J. Evol. Equ., 16(1):65-93, 2016.

[26] F. A. Williams. Combustion theory. Benjamin Cummings, second edition, 1985.

[27] C. Yu. Global weak solutions to the incompressible Navier-Stokes-Vlasov equations. J. Math. Pures Appl. (9), 100(2):275-293, 2013. 